\section{$\begin{array}{llllll}\text { I } & \mathbf{N} & \mathbf{F} & \mathbf{S} & \mathbf{Y} & \mathbf{S}\end{array}$ R E S E A R C H \\ $\begin{array}{llllll}\mathbf{R} & \mathrm{E} & \mathrm{P} & \mathrm{O} & \mathrm{R} & \mathrm{T}\end{array}$} P

Institut für Informationssysteme Abtg. Wissensbasierte Systeme Technische Universität Wien Favoritenstraße 9-11 A-1040 Wien, Austria Tel: $\quad+43-1-58801-18405$ Fax: $+43-1-58801-18493$ sek@kr.tuwien.ac.at www.kr.tuwien.ac.at

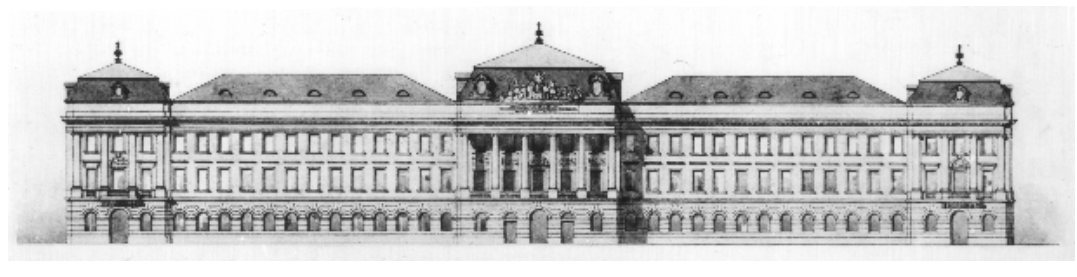

INSTITUT FÜR INFORMATIONSSYSTEME

AbTeilung Wissensbasierte Systeme

Probabilistic Logic Under Coherence, Model-Theoretic Probabilistic Logic, And Default Reasoning in System $P$

\author{
Veronica BIAZZO \\ Angelo GILIO \\ Thomas LUKASIEWICZ Giuseppe SANFILIPPO
}

INFSYS RESEARCH REPORT 1843-01-03

APRIL 2001; MAY 2002

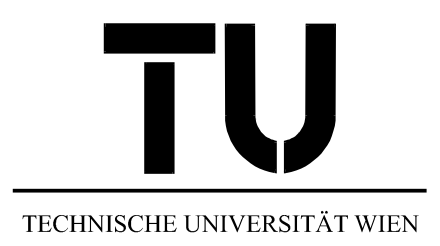



INFSYS RESEARCH REPORT

INFSYS RESEARCH REPORT 1843-01-03, APRIL 2001; MAY 2002

\title{
Probabilistic Logic under Coherence, Model-Theoretic Probabilistic Logic, And Default Reasoning in System $P$
}

\author{
Veronica Biazzo ${ }^{1}$, Angelo Gilio ${ }^{2}$, Thomas Lukasiewicz ${ }^{3}$, and Giuseppe Sanfilippo ${ }^{1}$
}

\begin{abstract}
We study probabilistic logic under the viewpoint of the coherence principle of de Finetti. In detail, we explore how probabilistic reasoning under coherence is related to model-theoretic probabilistic reasoning and to default reasoning in System $P$. In particular, we show that the notions of g-coherence and of g-coherent entailment can be expressed by combining notions in model-theoretic probabilistic logic with concepts from default reasoning. Moreover, we show that probabilistic reasoning under coherence is a generalization of default reasoning in System $P$. That is, we provide a new probabilistic semantics for System $P$, which neither uses infinitesimal probabilities nor atomic bound (or big-stepped) probabilities. These results also provide new algorithms for probabilistic reasoning under coherence and for default reasoning in System $P$, and they give new insight into default reasoning with conditional objects.
\end{abstract}

\footnotetext{
${ }^{1}$ Dipartimento di Matematica e Informatica, Università degli Studi di Catania, Città Universitaria, Viale A. Doria 6, 95152 Catania, Italy; email: \{vbiazzo, gsanfilippo\} @ dmi.unict.it.

${ }^{2}$ Dipartimento di Metodi e Modelli Matematici, Università di Roma “La Sapienza”, Via A. Scarpa 16, 00161 Roma, Italy; email: gilio@dmmm.uniroma1.it.

${ }^{3}$ Dipartimento di Informatica e Sistemistica, Università di Roma "La Sapienza", Via Salaria 113, 00198 Roma, Italy; email: lukasiew@dis.uniroma1.it. Alternate address: Institut für Informationssysteme, Technische Universität Wien, Favoritenstraße 9-11, 1040 Wien, Austria; email: lukasiew@kr.tuwien.ac.at.
}

Acknowledgements: This work has been partially supported by a DFG grant, a European Community Marie Curie Individual Fellowship (Disclaimer: The authors are solely responsible for information communicated and the European Commission is not responsible for any views or results expressed), and the Austrian Science Fund Project N Z29-INF. We are thankful to the reviewers of the ECSQARU-2001 version of this paper, whose constructive comments helped to improve this paper.

Copyright (c) 2002 by the authors 


\section{Introduction}

The probabilistic treatment of uncertainty plays an important role in many applications of knowledge representation and reasoning. Often, we need to reason with uncertain information under partial knowledge and then the use of precise probabilistic assessments seems unrealistic. Moreover, the family of uncertain quantities at hand has often no particular algebraic structure.

In such cases, a general approach is obtained by using (conditional and/or unconditional) probabilistic constraints, based on the coherence principle of de Finetti and suitable generalizations of it $[5,8,9,10$, $11,20,21,22,37]$, or on similar principles that have been adopted for lower and upper probabilities [36, 41]. Two important aspects in dealing with uncertainty are: (i) checking the consistency of a probabilistic assessment, and (ii) the propagation of a given assessment to further uncertain quantities.

Another approach for handling probabilistic constraints is model-theoretic probabilistic logic, whose roots go back to Boole's book of 1854 "The Laws of Thought" [7]. There is a wide spectrum of formal languages that have been explored in probabilistic logic, which ranges from constraints for unconditional and conditional events $[2,15,27,28,29,32]$ to rich languages that specify linear inequalities over events [14]. The main problems related to model-theoretic probabilistic logic are checking satisfiability, deciding logical consequence, and computing tight logically entailed intervals.

Coherence-based and model-theoretic probabilistic reasoning have been explored quite independently from each other by two different research communities. For this reason, the relationship between the two areas has not been studied in depth so far. The current paper and our work in [6] aim at filling this gap. More precisely, our research is essentially guided by the following two questions:

- Which is the semantic relationship between probabilistic reasoning under coherence and modeltheoretic probabilistic reasoning?

- Is it possible to use algorithms that have been developed for efficient reasoning in one area also in the other area?

Interestingly, it turns out that the answers to these two questions are closely related to default reasoning from conditional knowledge bases in System $P$. The literature contains several different proposals for default reasoning and extensive work on its desired properties. The core of these properties are the rationality postulates of System $P$ proposed by Kraus, Lehmann, and Magidor [25]. It turned out that these rationality postulates constitute a sound and complete axiom system for several classical model-theoretic entailment relations under uncertainty measures on worlds. More precisely, they characterize classical model-theoretic entailment under preferential structures [38, 25], infinitesimal probabilities [1,34], possibility measures [12], and world rankings [40,24]. They also characterize an entailment relation based on conditional objects [13]. A survey of all these relationships is given in $[3,16]$.

In this paper, we show that probabilistic reasoning under coherence is reducible to model-theoretic probabilistic reasoning using concepts from default reasoning. Crucially, we even show that probabilistic reasoning under coherence is a generalization of default reasoning in System $P$. That is, we give a new probabilistic semantics for System $P$, which neither uses infinitesimal probabilities [1,34] nor atomic bound [39] (or big-stepped [4]) probabilities. This paper deals with the semantic aspects of these findings, while the companion paper [6] focuses on its computational implications for probabilistic reasoning under coherence.

The main contributions of this paper can be summarized as follows:

- We define a coherence-based probabilistic logic. In particular, we define a formal language of logical and conditional constraints, which are defined on arbitrary families of conditional events. We then 
define the concepts of generalized coherence (or simply g-coherence) and of g-coherent entailment for this language.

- We explore the relationship between coherence-based and model-theoretic probabilistic reasoning. It turns out that the former generalizes default reasoning in System $P$, while the latter generalizes classical reasoning in propositional logic. Thus, the former does not have the property of inheritance of logical knowledge, while the latter does. We also show that g-coherent entailment coincides with logical entailment from a smaller knowledge base. Moreover, g-coherence lies between satisfiability and strong satisfiability, while g-coherent entailment is weaker than logical entailment.

- We show that g-coherence and g-coherent entailment can be reduced to the existence of satisfying probabilistic interpretations and to logical entailment, respectively, using concepts from default reasoning. Hence, these results provide new algorithms for coherence-based probabilistic reasoning, which are based on reductions to standard reasoning tasks in model-theoretic probabilistic logic.

- We explore the relationship between probabilistic reasoning under coherence and default reasoning in System $P$. We show that g-coherence and g-coherent entailment are generalizations of consistency and entailment in System $P$, respectively. Hence, we provide a new probabilistic semantics for System $P$. Moreover, these results also provide a new algorithm for default reasoning in System $P$.

- We provide new insight into Dubois and Prade's approach to default reasoning with conditional objects $[13,3]$. In detail, we show that the definitions of consistency and entailment for conditional objects are actually classical counterparts of the definitions of g-coherence and g-coherent entailment, respectively.

The rest of this paper is organized as follows. Section 2 introduces the formal background of this work. In Section 3, we explore the relationship between probabilistic reasoning under coherence and modeltheoretic probabilistic reasoning. Sections 4 and 5 focus on the relationship between probabilistic reasoning under coherence and default reasoning in System $P$ and with conditional objects, respectively. In Section 6 , we summarize the main results and give an outlook on future research. Note that detailed proofs of all results are given in Appendices A-C.

\section{Probabilistic logic under coherence}

In this section, we first introduce some technical preliminaries. We then briefly describe precise and imprecise probability assessments under coherence. We finally define our coherence-based probabilistic logic and give an illustrating example.

\subsection{Preliminaries}

We first define the syntax and semantics of events. We assume a nonempty set of basic events $\Phi$. We use $\perp$ and $T$ to denote false and true, respectively. The set of events is the closure of $\Phi \cup\{\perp, \top\}$ under the Boolean operators $\neg$ and $\wedge$. That is, each element of $\Phi \cup\{\perp, \top\}$ is an event, and if $\phi$ and $\psi$ are events, then also $(\phi \wedge \psi)$ and $\neg \phi$. We use $(\phi \vee \psi)$ and $(\psi \Leftarrow \phi)$ to abbreviate $\neg(\neg \phi \wedge \neg \psi)$ and $\neg(\phi \wedge \neg \psi)$, respectively, and adopt the usual conventions to eliminate parentheses. A logical constraint is an event of the form $\psi \Leftarrow \phi$. Note that $\perp \Leftarrow \alpha$ is equivalent to $\neg \alpha$. 
A world $I$ is a truth assignment to the basic events in $\Phi$ (that is, a mapping $I: \Phi \rightarrow\{$ false, true $\}$ ), which is extended to all events as usual (that is, $(\phi \wedge \psi)$ is true in $I$ iff $\phi$ and $\psi$ are true in $I$, and $\neg \phi$ is true in $I$ iff $\phi$ is not true in $I$ ). Denote by $\mathcal{I}_{\Phi}$ the set of all worlds for $\Phi$. We often identify the truth values false and true with the real numbers 0 and 1, respectively. A world $I$ satisfies an event $\phi$, or $I$ is a model of $\phi$, denoted $I \models \phi$, iff $I(\phi)=$ true. I satisfies a set of events $L$, or $I$ is a model of $L$, denoted $I \models L$, iff $I$ is a model of all $\phi \in L$. An event $\phi$ (resp., a set of events $L$ ) is satisfiable iff a model of $\phi$ (resp., $L$ ) exists. An event $\psi$ is a logical consequence of $\phi$ (resp., $L$ ), denoted $\phi \models \psi$ (resp., $L \models \psi$ ), iff each model of $\phi$ (resp., $L$ ) is also a model of $\psi$. We use $\phi \not \models \psi$ (resp., $L \not \models \psi$ ) to denote that $\phi \models \psi$ (resp., $L \models \psi$ ) does not hold.

\subsection{Probability assessments}

We next define conditional events, (precise) probability assessments on conditional events, and the notion of coherence for such probability assessments.

A conditional event is an expression of the form $\psi \mid \phi$ with events $\psi$ and $\phi$. It can be looked at as a threevalued logical entity, with values true, or false, or indeterminate, according to whether $\psi$ and $\phi$ are true, or $\psi$ is false and $\phi$ is true, or $\phi$ is false, respectively. That is, we extend worlds $I$ to conditional events $\psi \mid \phi$ by $I(\psi \mid \phi)=$ true iff $I \models \psi \wedge \phi, I(\psi \mid \phi)=$ false iff $I \mid=\neg \psi \wedge \phi$, and $I(\psi \mid \phi)=$ indeterminate iff $I \models \neg \phi$. Note that $\psi \mid \phi$ coincides with $\psi \wedge \phi \mid \phi$. More generally, $\psi_{1} \mid \phi_{1}$ and $\psi_{2} \mid \phi_{2}$ coincide iff $\psi_{1} \wedge \phi_{1}=$ $\psi_{2} \wedge \phi_{2}$ and $\phi_{1}=\phi_{2}$.

We recall that in the framework of subjective probability, given an event $\psi$, the evaluation $\operatorname{Pr}(\psi)=\alpha$, given by someone, is a numerical representation of his degree of belief on $\psi$ being true. Using the betting criterion, if such individual evaluates $\operatorname{Pr}(\psi)=\alpha$, then he will pay an amount of money $\alpha S$, where $S \neq 0$ is arbitrary, and he will get back the amount $S \psi$. Then, the associated random gain is $G=S(\psi-\alpha)$. Coherence requires that, for every $S \neq 0$, it must be $\max G \geq 0$, which implies $0 \leq \alpha \leq 1$.

For a conditional event $\psi \mid \phi$, if the individual evaluates $\operatorname{Pr}(\psi \mid \phi)=\alpha$, then he will pay an amount of money $\alpha S$ getting back the amount $S \psi$ (resp., $\alpha S$ ) if $\phi$ is true (resp., false). Then, the associated random gain is $G=S \phi(\psi-\alpha)$. Coherence requires that, for every $S \neq 0$, it must be max $G \mid \phi \geq 0$, which (again) implies $0 \leq \alpha \leq 1$.

We point out that the coherence-based probabilistic approach is more general than the usual one, because to assess conditional probabilities we do not rely on unconditional probabilities. In fact, in our framework the probability assessment $\operatorname{Pr}(\psi \mid \phi)=\alpha$ has a natural meaning in all cases, including the one in which $\operatorname{Pr}(\phi)=0$. In other words, the quantity $\operatorname{Pr}(\phi)$ plays no role and the only relevant thing is the assumption " $\phi$ true". Moreover, given a function $A$ on a family of conditional events $\mathcal{C}$, if $A$ is coherent, then $A$ satisfies all the usual axioms of a conditional probability, while the converse is not true (for a counterexample see [19], Example 8).

More formally, a probability assessment $(L, A)$ on a set of conditional events $\mathcal{C}$ consists of a set of logical constraints $L$, and a mapping $A$ that assigns each $c \in \mathcal{C}$ a real number in $[0,1]$. Informally, $L$ describes logical relationships, while $A$ represents probabilistic knowledge. For $\left\{\psi_{1}\left|\phi_{1}, \ldots, \psi_{n}\right| \phi_{n}\right\} \subseteq \mathcal{C}$ with $n \geq 1$ and $n$ real numbers $s_{1}, \ldots, s_{n}$, let the mapping $G: \mathcal{I}_{\Phi} \rightarrow \mathbf{R}$ be defined as follows. For every $I \in \mathcal{I}_{\Phi}$ :

$$
G(I)=\sum_{i=1}^{n} s_{i} \cdot I\left(\phi_{i}\right) \cdot\left(I\left(\psi_{i}\right)-A\left(\psi_{i} \mid \phi_{i}\right)\right) .
$$

In the framework of betting criterion, $G$ can be interpreted as the random gain corresponding to a combination of $n$ bets of amounts $s_{1} \cdot A\left(\psi_{1} \mid \phi_{1}\right), \ldots, s_{n} \cdot A\left(\psi_{n} \mid \phi_{n}\right)$ on $\psi_{1}\left|\phi_{1}, \ldots, \psi_{n}\right| \phi_{n}$ with stakes $s_{1}, \ldots, s_{n}$. In detail, to bet on $\psi_{i} \mid \phi_{i}$, one pays an amount of $s_{i} \cdot A\left(\psi_{i} \mid \phi_{i}\right)$, and one gets back the amount of $s_{i}, 0$, 
and $s_{i} \cdot A\left(\psi_{i} \mid \phi_{i}\right)$, when $\psi_{i} \wedge \phi_{i}, \neg \psi_{i} \wedge \phi_{i}$, and $\neg \phi_{i}$, respectively, turns out to be true. The following notion of coherence now assures that it is impossible (for both the gambler and the bookmaker) to have uniform loss. A probability assessment $(L, A)$ on a set of conditional events $\mathcal{C}$ is coherent iff for every $\left\{\psi_{1}\left|\phi_{1}, \ldots, \psi_{n}\right| \phi_{n}\right\} \subseteq \mathcal{C}$ with $n \geq 1$ and for all real numbers $s_{1}, \ldots, s_{n}$, it holds $\max \left\{G(I) \mid I \in \mathcal{I}_{\Phi}, I \models L\right.$, $\left.I \models \phi_{1} \vee \cdots \vee \phi_{n}\right\} \geq 0$.

\subsection{Imprecise probability assessments}

We now describe imprecise probability assessments on conditional events and the concepts of g-coherence and g-coherent entailment for such assessments.

An imprecise probability assessment $(L, A)$ on a set of conditional events $\mathcal{C}$ consists of a set of logical constraints $L$ and a mapping $A$ that assigns each $c \in \mathcal{C}$ an interval $[l, u] \subseteq[0,1]$ with $l \leq u$. We say $(L, A)$ is $g$-coherent iff there exists a coherent precise probability assessment $\left(L, A^{\star}\right)$ on $\mathcal{C}$ such that $A^{\star}(c) \in A(c)$ for all $c \in \mathcal{C}$.

We recall a characterization of g-coherence due to Gilio [20]; equivalent results have been obtained by Coletti [8]. Given a set of logical constraints $L$ and a set of conditional events $\mathcal{C}=\left\{c_{1}, \ldots, c_{n}\right\}$, denote by $R_{L}(\mathcal{C})$ the set of all mappings $r$ that assign each $c_{i}=\psi_{i} \mid \phi_{i} \in \mathcal{C}$ a member of $\left\{\psi_{i} \wedge \phi_{i}, \neg \psi_{i} \wedge \phi_{i}, \neg \phi_{i}\right\}$ such that $(i) L \cup\left\{r\left(c_{i}\right) \mid c_{i} \in \mathcal{C}\right\}$ is satisfiable, and $(i i) r\left(c_{i}\right) \neq \neg \phi_{i}$ for some $i \in\{1, \ldots, n\}$. For such mappings $r$ and events $\phi$, we use $r \models \phi$ to abbreviate $r\left(c_{1}\right) \wedge \cdots \wedge r\left(c_{n}\right) \models \phi$.

Theorem 2.1 (Gilio [20]) An imprecise probability assessment $(L, A)$ on a set of conditional events $\mathcal{C}$ is $g$ coherent iff for every $\mathcal{C}_{n}=\left\{\psi_{1}\left|\phi_{1}, \ldots, \psi_{n}\right| \phi_{n}\right\} \subseteq \mathcal{C}$ with $n \geq 1$, the following system of linear constraints over the variables $\lambda_{r}(r \in R)$, where $R=R_{L}\left(\mathcal{C}_{n}\right)$, is solvable:

$$
\begin{aligned}
\sum_{r \in R} \lambda_{r} \cdot p_{r i} & \geq l_{i} \quad(\text { for all } i \in\{1, \ldots, n\}) \\
\sum_{r \in R} \lambda_{r} \cdot q_{r i} & \leq u_{i} \quad(\text { for all } i \in\{1, \ldots, n\}) \\
\sum_{r \in R} \lambda_{r} & =1 \\
\lambda_{r} & \geq 0 \quad(\text { for all } r \in R),
\end{aligned}
$$

where $l_{i}$ and $u_{i}$ are defined by $A\left(\psi_{i} \mid \phi_{i}\right)=\left[l_{i}, u_{i}\right]$ for all $i \in\{1, \ldots, n\}$, and $p_{r i}$ and $q_{r i}$ are defined as follows for all $r \in R$ and $i \in\{1, \ldots, n\}$ :

$$
\left.p_{r i} \text { (resp., } q_{r i}\right)= \begin{cases}1, & \text { if } r \models \psi_{i} \wedge \phi_{i} \\ 0, & \text { if } r \models \neg \psi_{i} \wedge \phi_{i} \\ l_{i}\left(\text { resp., } u_{i}\right), & \text { if } r \models \neg \phi_{i} .\end{cases}
$$

We next define the notion of g-coherent entailment for imprecise probability assessments. Let $(L, A)$ be a g-coherent imprecise probability assessment on a set of conditional events $\mathcal{C}$. The imprecise probability assessment $[l, u]$ on a conditional event $\gamma$ is called a $g$-coherent consequence of $(L, A)$ iff $A^{\star}(\gamma) \in[l, u]$ for every g-coherent precise probability assessment $A^{\star}$ on $\mathcal{C} \cup\{\gamma\}$ such that $A^{\star}(c) \in A(c)$ for all $c \in \mathcal{C}$. It is a tight $g$-coherent consequence of $(L, A)$ iff $l$ (resp., $u)$ is the infimum (resp., supremum) of $A^{\star}(\gamma)$ subject to all g-coherent precise probability assessments $A^{\star}$ on $\mathcal{C} \cup\{\gamma\}$ such that $A^{\star}(c) \in A(c)$ for all $c \in \mathcal{C}$. Observe that for $c=\beta \mid \alpha$ such that $L \models \neg \alpha$, every $\{(c,[l, u])\}$ with $l, u \in[0,1]$ is a g-coherent consequence of $(L, A)$, and $\{(c,[1,0])\}$ is the unique tight g-coherent consequence of $(L, A)$. Note that here we identify $[1,0]$ with the empty set. 


\subsection{Probabilistic logic under coherence}

We now define conditional constraints, probabilistic knowledge bases, and the notions of g-coherence and g-coherent entailment for probabilistic knowledge bases. In the rest of this paper, we assume that $\Phi$ is finite.

A conditional constraint is an expression of the form $(\psi \mid \phi)[l, u]$ with real numbers $l, u \in[0,1]$ and events $\psi, \phi$. We call $\phi$ its antecedent and $\psi$ its consequent. A probabilistic knowledge base $K B=(L, P)$ consists of a finite set of logical constraints $L$, and a finite set of conditional constraints $P$ such that (i) $l \leq u$ for all $(\psi \mid \phi)[l, u] \in P$, and (ii) $\psi_{1}\left|\phi_{1} \neq \psi_{2}\right| \phi_{2}$ for any two distinct $\left(\psi_{1} \mid \phi_{1}\right)\left[l_{1}, u_{1}\right],\left(\psi_{2} \mid \phi_{2}\right)\left[l_{2}, u_{2}\right] \in P$.

Every imprecise probability assessment $I P=(L, A)$ with finite $L$ on a finite set of conditional events $\mathcal{C}$ can be represented by the probabilistic knowledge base

$$
K B_{I P}=(L,\{(\psi \mid \phi)[l, u]|\psi| \phi \in \mathcal{C}, A(\psi \mid \phi)=[l, u]\}) .
$$

Conversely, every probabilistic knowledge base $K B=(L, P)$ can be expressed by the following imprecise probability assessment $I P_{K B}=\left(L, A_{K B}\right)$ on $\mathcal{C}_{K B}$ :

$$
\begin{aligned}
& A_{K B}=\{(\psi \mid \phi,[l, u]) \mid(\psi \mid \phi)[l, u] \in K B\}, \\
& \mathcal{C}_{K B}=\{\psi|\phi| \exists l, u \in[0,1]:(\psi \mid \phi)[l, u] \in K B\} .
\end{aligned}
$$

A probabilistic knowledge base $K B$ is said g-coherent iff $I P_{K B}$ is g-coherent. For g-coherent $K B$ and conditional constraints $(\psi \mid \phi)[l, u]$, we say $(\psi \mid \phi)[l, u]$ is a g-coherent consequence of $K B$, denoted $K B \sim(\psi \mid \phi)[l, u]$, iff $\{(\psi \mid \phi,[l, u])\}$ is a g-coherent consequence of $I P_{K B}$. It is a tight g-coherent consequence of $K B$, denoted $K B \sim_{\text {tight }}(\psi \mid \phi)[l, u]$, iff $\{(\psi \mid \phi,[l, u])\}$ is a tight g-coherent consequence of $I P_{K B}$.

We give an example to illustrate the concept of a probabilistic knowledge base and the notions of $\mathrm{g}$ coherence and of g-coherent entailment.

Example 2.2 Consider the following probabilistic knowledge base $K B=(L, P)$ :

$$
\begin{aligned}
& L=\{\text { bird } \Leftarrow \text { penguin }\} \\
& P=\{(\text { legs } \mid \text { bird })[1,1],(\text { fly } \mid \text { bird })[1,1],(\text { fly } \mid \text { penguin })[0, .05]\} .
\end{aligned}
$$

In probabilistic logic under coherence, $K B$ may represent the logical knowledge "all penguins are birds", the logical default knowledge "generally, birds have legs" and "generally, birds fly", and the probabilistic default knowledge "generally, penguins fly with a probability of at most 0.05 ". It is not difficult to see that $K B$ is g-coherent, and that some tight g-coherent consequences of $K B$ are given as follows:

$$
\begin{aligned}
& K B \sim_{\text {tight }}(\text { legs } \mid \text { bird })[1,1], \quad K B \sim_{\text {tight }}(\text { fly } \mid \text { bird })[1,1] \\
& K B \sim_{\text {tight }}(\text { legs } \mid \text { penguin })[0,1], \quad K B \sim_{\text {tight }}(\text { fly } \mid \text { penguin })[0, .05]
\end{aligned}
$$

Here, the interval " $[0,1]$ " is due to the fact that the logical property of having legs is not inherited from birds down to penguins.

\section{Relationship to model-theoretic probabilistic logic}

In this section, we explore the relationship between probabilistic logic under coherence and model-theoretic probabilistic logic. We first briefly recall the main concepts of model-theoretic probabilistic logic. We then show how the notion of g-coherence can be reduced to the existence of satisfying probabilistic interpretations 
in model-theoretic probabilistic logic. Moreover, we show that g-coherent entailment can be reduced to logical entailment in model-theoretic probabilistic logic. We finally describe the rough relationship between g-coherence and satisfiability (resp., strong satisfiability), and between g-coherent entailment and logical entailment.

\subsection{Model-theoretic probabilistic logic}

We now briefly recall the main concepts of model-theoretic probabilistic logic. In particular, we define probabilistic interpretations and the semantics of events, logical constraints, and conditional constraints under probabilistic interpretations. We then define the notions of satisfiability and logical entailment.

A probabilistic interpretation $\operatorname{Pr}$ is a probability function on $\mathcal{I}_{\Phi}$ (that is, a mapping $\operatorname{Pr}: \mathcal{I}_{\Phi} \rightarrow[0,1]$ such that all $\operatorname{Pr}(I)$ with $I \in \mathcal{I}_{\Phi}$ sum up to 1). The probability of an event $\phi$ in the probabilistic interpretation $\operatorname{Pr}$, denoted $\operatorname{Pr}(\phi)$, is defined as the sum of all $\operatorname{Pr}(I)$ such that $I \in \mathcal{I}_{\Phi}$ and $I=\phi$. For events $\phi$ and $\psi$ with $\operatorname{Pr}(\phi)>0$, we use $\operatorname{Pr}(\psi \mid \phi)$ to abbreviate $\operatorname{Pr}(\psi \wedge \phi) / \operatorname{Pr}(\phi)$. The truth of logical and conditional constraints $F$ in a probabilistic interpretation $\operatorname{Pr}$, denoted $\operatorname{Pr} \models F$, is defined by:

- $\operatorname{Pr} \models \psi \Leftarrow \phi$ iff $\operatorname{Pr}(\psi \wedge \phi)=\operatorname{Pr}(\phi)$.

- $\operatorname{Pr} \models(\psi \mid \phi)[l, u]$ iff $\operatorname{Pr}(\phi)=0$ or $\operatorname{Pr}(\psi \mid \phi) \in[l, u]$.

We say $\operatorname{Pr}$ satisfies a logical or conditional constraint $F$, or $\operatorname{Pr}$ is a model of $F$, iff $\operatorname{Pr}=F$. We say $\operatorname{Pr}$ satisfies a set of logical and conditional constraints $\mathcal{F}$, or $\operatorname{Pr}$ is a model of $\mathcal{F}$, denoted $\operatorname{Pr} \models \mathcal{F}$, iff $\operatorname{Pr}$ is a model of all $F \in \mathcal{F}$. We say $\mathcal{F}$ is satisfiable iff a model of $\mathcal{F}$ exists. We say $\mathcal{F}$ is strongly satisfiable iff a model $\operatorname{Pr}$ of $\mathcal{F}$ exists such that $\operatorname{Pr}(\phi)>0$ for all $(\psi \mid \phi)[l, u] \in \mathcal{F}$.

We next define the notion of logical entailment. A conditional constraint $F=(\psi \mid \phi)[l, u]$ is a logical consequence of a set of logical and conditional constraints $\mathcal{F}$, denoted $\mathcal{F}=F$, iff each model of $\mathcal{F}$ is also a model of $F$. It is a tight logical consequence of $\mathcal{F}$, denoted $\left.\mathcal{F}\right|_{\text {tight }} F$, iff $l$ (resp., $u$ ) is the infimum (resp., supremum) of $\operatorname{Pr}(\psi \mid \phi)$ subject to all models $\operatorname{Pr}$ of $\mathcal{F}$ with $\operatorname{Pr}(\phi)>0$. Note that we define $l=1$ and $u=0$, when $\mathcal{F} \models(\phi \mid \top)[0,0]$. A probabilistic knowledge base $K B=(L, P)$ is satisfiable (resp., strongly satisfiable) iff $L \cup P$ is satisfiable (resp., strongly satisfiable). A conditional constraint $(\psi \mid \phi)[l, u]$ is a logical consequence of $K B$, denoted $K B=(\psi \mid \phi)[l, u]$, iff $L \cup P \models(\psi \mid \phi)[l, u]$. It is a tight logical consequence of $K B$, denoted $K B \models_{\text {tight }}(\psi \mid \phi)[l, u]$, iff $L \cup P \models_{\text {tight }}(\psi \mid \phi)[l, u]$.

Example 3.1 Consider again the probabilistic knowledge base $K B=(L, P)$ of Example 2.2. In modeltheoretic probabilistic logic, $K B$ may represent the logical knowledge "all penguins are birds", "all birds have legs", and "all birds fly" (that is, in model-theoretic probabilistic logic, a logical constraint $\psi \Leftarrow \phi$ has the same meaning as the conditional constraint $(\psi \mid \phi)[1,1]$ ), and the probabilistic knowledge "penguins fly with a probability of at most 0.05 ". It is easy to see that $K B$ is satisfiable, but not strongly satisfiable. Some tight logical consequences of $K B$ are given as follows:

$$
\begin{aligned}
& K B \models_{\text {tight }}(\text { legs } \mid \text { bird })[1,1], K B \models_{\text {tight }}(\text { fly } \mid \text { bird })[1,1], \\
& K B \models_{\text {tight }} \text { (legs|penguin) }[1,0], K B \models_{\text {tight }}(\text { fly } \mid \text { penguin })[1,0] .
\end{aligned}
$$

Here, we have the empty set "[ $[1,0]$ " in the last two conditional constraints, as the logical property of being able to fly is inherited from birds down to penguins, and is then incompatible there with "penguins fly with a probability of at most 0.05 ". 


\subsection{G-coherence in model-theoretic probabilistic logic}

The following theorem shows how g-coherence can be expressed through the existence of satisfying probabilistic interpretations. This result follows from Theorem 2.1. Roughly, it says that $K B=(L, P)$ is gcoherent iff every nonempty $P^{\prime} \subseteq P$ has a model $\operatorname{Pr}$ such that $\operatorname{Pr}=L$ and that $\operatorname{Pr}(\phi)>0$ for at least one $(\psi \mid \phi)[l, u] \in P^{\prime}$. Hence, if $K B$ is g-coherent, then $K B$ is also satisfiable, that is, $L \cup P$ has a model, or equivalently every nonempty $P^{\prime} \subseteq P$ has a model $\operatorname{Pr}$ such that $\operatorname{Pr} \models L$.

Theorem 3.2 Let $K B=(L, P)$ be a probabilistic knowledge base. Then, $K B$ is g-coherent iff for every nonempty $P_{n}=\left\{\left(\psi_{1} \mid \phi_{1}\right)\left[l_{1}, u_{1}\right], \ldots,\left(\psi_{n} \mid \phi_{n}\right)\left[l_{n}, u_{n}\right]\right\} \subseteq P$, there exists a model $\operatorname{Pr}$ of $L \cup P_{n}$ such that $\operatorname{Pr}\left(\phi_{1} \vee \cdots \vee \phi_{n}\right)>0$.

The next theorem shows that g-coherence has a characterization similar to the one of $p$-consistency in default reasoning by Goldszmidt and Pearl [23]. It follows from Theorem 3.2. To formulate this result, we adopt the following terminology from default reasoning from conditional knowledge bases [16, 3]. A probabilistic interpretation $\operatorname{Pr}$ verifies a conditional constraint $(\psi \mid \phi)[l, u]$ iff $\operatorname{Pr}(\phi)>0$ and $\operatorname{Pr}=(\psi \mid \phi)[l, u]$. A set of conditional constraints $P$ tolerates a conditional constraint $F$ under a set of logical constraints $L$ iff there exists a model of $L \cup P$ that verifies $F$. We say $P$ is under $L$ in conflict with $F$ iff no model of $L \cup P$ verifies $F$.

Theorem 3.3 A probabilistic knowledge base $K B=(L, P)$ is g-coherent iff there exists an ordered partition $\left(P_{0}, \ldots, P_{k}\right)$ of $P$ such that either

(a) every $P_{i}, 0 \leq i \leq k$, is the set of all $F \in \bigcup_{j=i}^{k} P_{j}$ tolerated under $L$ by $\bigcup_{j=i}^{k} P_{j}$, or

(b) for every $i, 0 \leq i \leq k$, each $F \in P_{i}$ is tolerated under $L$ by $\bigcup_{j=i}^{k} P_{j}$.

In a companion paper [6], we use Theorem 3.3 to give a new algorithm for deciding g-coherence, which is essentially a reformulation of a previous algorithm by Gilio [20] using terminology from default reasoning, and which is closely related to an algorithm for checking $p$-consistency by Goldszmidt and Pearl [23]. ${ }^{1}$

\subsection{G-coherent entailment in model-theoretic probabilistic logic}

We now show that g-coherent entailment can be reduced to logical entailment. We first give some preparative definitions.

For probabilistic knowledge bases $K B=(L, P)$ and events $\alpha$ such that $L \not \models \neg \alpha$, let $P_{\alpha}(K B)$ denote the set of all subsets $P_{n}=\left\{\left(\psi_{1} \mid \phi_{1}\right)\left[l_{1}, u_{1}\right], \ldots,\left(\psi_{n} \mid \phi_{n}\right)\left[l_{n}, u_{n}\right]\right\}$ of $P$ such that every model $\operatorname{Pr}$ of $L \cup P_{n}$ with $\operatorname{Pr}\left(\phi_{1} \vee \cdots \vee \phi_{n} \vee \alpha\right)>0$ satisfies $\operatorname{Pr}(\alpha)>0$. For $K B=(L, P)$ and $\alpha$ with $L \models \neg \alpha$, let $P_{\alpha}(K B)=\{\emptyset\}$.

The following theorem shows that the tight interval entailed under g-coherence can be expressed as the intersection of some logically entailed tight intervals. It says that $(\beta \mid \alpha)[l, u]$ is a tight g-coherent consequence of $K B=(L, P)$ iff $[l, u]$ is the intersection of all $[c, d]$ such that $L \cup P^{\prime} F_{\text {tight }}(\beta \mid \alpha)[c, d]$ for some $P^{\prime} \in P_{\alpha}(K B)$. This follows from Theorem 3.2, which implies that the tight interval $[l, u]$ entailed under g-coherence is the intersection of all $[c, d]$ such that $c$ (resp., $d$ ) is the infimum (resp., supremum) of $p$ subject to $\operatorname{Pr} \models L \cup P_{n} \cup\{(\beta \mid \alpha)[p, p]\}$ and $\operatorname{Pr}\left(\phi_{1} \vee \cdots \vee \phi_{n} \vee \alpha\right)>0$ for some $P_{n}=\left\{\left(\psi_{1} \mid \phi_{1}\right)\left[l_{1}, u_{1}\right], \ldots\right.$, $\left.\left(\psi_{n} \mid \phi_{n}\right)\left[l_{n}, u_{n}\right]\right\} \subseteq P$.

\footnotetext{
${ }^{1}$ Note that the relationship between the algorithms in [20] and [23] was suggested first by Didier Dubois (personal communication).
} 
Theorem 3.4 Let $K B=(L, P)$ be a g-coherent probabilistic knowledge base, and let $\beta \mid \alpha$ be a conditional event. Then, $K B \sim_{\text {tight }}(\beta \mid \alpha)[l, u]$, where

$$
[l, u]=\bigcap\left\{[c, d] \mid L \cup P^{\prime} \models_{\text {tight }}(\beta \mid \alpha)[c, d] \text { for some } P^{\prime} \in P_{\alpha}(K B)\right\} .
$$

Clearly, this reduction of g-coherent entailment to logical entailment is computationally expensive, as we have to compute a tight logically entailed interval for each member of $P_{\alpha}(K B)$. In the following, we show that we can restrict our attention to the unique greatest element in $P_{\alpha}(K B)$ with respect to set inclusion. The following lemma shows that $P_{\alpha}(K B)$ contains indeed such a unique greatest element. This result can be proved by showing that $P_{\alpha}(K B)$ is nonempty and closed under set union.

Lemma 3.5 Let $K B=(L, P)$ be a g-coherent probabilistic knowledge base, and let $\alpha$ be an event. Then, $P_{\alpha}(K B)$ contains a unique greatest element.

The next theorem shows the crucial result that g-coherent entailment of $(\beta \mid \alpha)[l, u]$ from $K B$ can be reduced to logical entailment of $(\beta \mid \alpha)[l, u]$ from the greatest element in $P_{\alpha}(K B)$. It follows from Theorem 3.4 and Lemma 3.5, using the monotonicity of logical consequence (which says that, if $\mathcal{F} \models F$ and $\mathcal{F} \subseteq \mathcal{F}^{\prime}$, then $\mathcal{F}^{\prime} \models F$ ).

Theorem 3.6 Let $K B=(L, P)$ be a g-coherent probabilistic knowledge base, and let $F=(\beta \mid \alpha)[l, u]$ be a conditional constraint. Let $K B^{\star}=\left(L, P^{\star}\right)$, where $P^{\star}$ is the greatest element in $P_{\alpha}(K B)$. Then,

(a) $K B \sim F$ iff $K B^{\star} \models F$.

(b) $K B \sim_{\text {tight }} F$ iff $K B^{\star} \models_{\text {tight }} F$.

Thus, computing tight g-coherent consequences can be reduced to computing tight logical consequences from the greatest element $P^{\star}$ in $P_{\alpha}(K B)$. The following theorem shows how $P^{\star}$ can be characterized and thus computed. It specifies some $P^{\star}$ by two conditions (i) and (ii). It can then be shown that (i) implies that every member of $P_{\alpha}(K B)$ is a subset of $P^{\star}$, and that (ii) implies that $P^{\star}$ belongs to $P_{\alpha}(K B)$. In summary, this proves that the specified $P^{\star}$ is the greatest element in $P_{\alpha}(K B)$.

Theorem 3.7 Let $K B=(L, P)$ be a g-coherent probabilistic knowledge base, and let $\alpha$ be an event. Let $P^{\star} \subseteq P$, and let $\left(P_{0}, \ldots, P_{k}\right)$ be an ordered partition of $P \backslash P^{\star}$ such that the following two conditions (i) and (ii) hold:

(i) Every $P_{i}, 0 \leq i \leq k$, is the set of all elements in $P_{i} \cup \cdots \cup P_{k} \cup P^{\star}$ that are tolerated under $L \cup$ $\{\perp \Leftarrow \alpha\}$ by $P_{i} \cup \cdots \cup P_{k} \cup P^{\star}$.

(ii) No member of $P^{\star}$ is tolerated under $L \cup\{\perp \Leftarrow \alpha\}$ by $P^{\star}$.

Then, $P^{\star}$ is the greatest element in $P_{\alpha}(K B)$.

Hence, by Theorems 3.6 and 3.7, the tight interval under g-coherent entailment can be computed by first computing $P^{\star}$ by a g-coherence check, and then computing the tight interval under logical entailment from $P^{\star}$. This new algorithm for computing tight g-coherent consequences has been formulated in a companion paper [6].

Semantically, Theorems 3.6 and 3.7 draw a precise picture of the relationship between g-coherent entailment and logical entailment. They show that g-coherent entailment coincides with logical entailment from 
a smaller probabilistic knowledge base. That is, under g-coherent entailment, we simply cut away a part of the knowledge base. Roughly speaking, we remove every conditional constraint $(\psi \mid \phi)[l, u] \in P$ such that $\phi$ is "larger" than $\alpha$. Intuitively, g-coherent entailment does not have the property of inheritance, neither for logical knowledge nor for probabilistic knowledge, while logical entailment shows inheritance of logical knowledge, but not of probabilistic knowledge. The following example illustrates this difference.

Example 3.8 Consider the following probabilistic knowledge base:

$$
K B=(\{\text { bird } \Leftarrow \text { penguin }\},\{(\text { legs } \mid \text { bird })[1,1], \text { (wings } \mid \text { bird })[.95,1]\}) .
$$

Observe that $K B$ is g-coherent and strongly satisfiable. Notice then that by Theorem 3.6 the conditional constraint $(\psi \mid$ penguin) $[l, u]$ is a tight g-coherent consequence of $K B$ iff it is a tight logical consequence of $K B^{\star}=(\{$ bird $\Leftarrow$ penguin $\}, \emptyset)$. By this characterization, the following tight g-coherent consequences of $K B$ are immediate:

$$
K B \sim_{\text {tight }} \text { (legs|penguin) }[0,1] \text { and } K B \sim_{\text {tight }} \text { (wings|penguin) }[0,1] .
$$

Moreover, we have the following tight logical consequences of $K B$ :

$$
K B=_{\text {tight }} \text { (legs } \mid \text { penguin) }[1,1] \text { and } K B \models_{\text {tight }} \text { (wings } \mid \text { penguin) }[0,1] .
$$

In summary, under g-coherent entailment, neither the logical property of having legs nor the probabilistic one of having wings is inherited from birds to penguins. Under logical entailment, the logical property is inherited, while the probabilistic one is not.

\subsection{Coherence-based versus model-theoretic probabilistic logic}

We now describe the rough relationship between g-coherence and satisfiability (resp., strong satisfiability), and between g-coherent entailment and logical entailment. The following theorem shows that g-coherence implies satisfiability, and that strong satisfiability implies g-coherence. That is, g-coherence lies between satisfiability and strong satisfiability. These results are immediate by Theorem 3.2.

Theorem 3.9 (a) Every g-coherent probabilistic knowledge base KB is satisfiable.

\section{(b) Every strongly satisfiable probabilistic knowledge base KB is g-coherent.}

The following example shows that the converse statements generally do not hold. That is, strong satisfiability is strictly stronger than g-coherence, and g-coherence itself is strictly stronger than satisfiability.

Example 3.10 (a) The probabilistic knowledge base $K B=(\emptyset,\{($ fly $\mid$ bird $)[.9,1]$, ( $\neg$ fly $\mid$ bird $)[.2,1]\})$ is satisfiable, but not g-coherent.

(b) The probabilistic knowledge base $K B=(\{$ bird $\Leftarrow$ penguin $\},\{($ fly $\mid$ bird $)[1,1]$, $(\neg$ fly $\mid$ penguin $)[1,1]\})$ is g-coherent, but not strongly satisfiable.

The following theorem shows that logical entailment is stronger than g-coherent entailment. That is, g-coherent consequence implies logical consequence (or there are more conditional constraints logically entailed than entailed under g-coherence) and the tight intervals that are entailed under logical entailment are subintervals of those entailed under g-coherent entailment. This result is immediate by Theorem 3.6. 
Theorem 3.11 Let $K B=(L, P)$ be a g-coherent probabilistic knowledge base, and let $(\beta \mid \alpha)[l, u]$ and $(\beta \mid \alpha)[r, s]$ be two conditional constraints. Then,

(a) $K B \sim(\beta \mid \alpha)[l, u]$ implies $K B \models(\beta \mid \alpha)[l, u]$.

(b) $K B \sim_{\text {tight }}(\beta \mid \alpha)[l, u]$ and $K B \models_{\text {tight }}(\beta \mid \alpha)[r, s]$ implies $[l, u] \supseteq[r, s]$.

The next example shows that logical entailment is in fact strictly stronger than g-coherent entailment (recall that we identify $[1,0]$ with the empty set).

Example 3.12 Consider the following probabilistic knowledge bases $K B_{1}$ and $K B_{2}$ :

$$
\begin{aligned}
& K B_{1}=(\emptyset,\{(\text { fly } \mid \text { bird })[1,1],(\text { mobile } \mid \text { fly })[1,1]\}), \\
& K B_{2}=(\emptyset,\{(\text { fly } \mid \text { bird })[1,1],(\text { bird } \mid \text { penguin })[1,1],(\neg \text { fly } \mid \text { penguin })[1,1]\}) .
\end{aligned}
$$

It is not difficult to see that $K B_{1}$ and $K B_{2}$ are both g-coherent and satisfiable. Some tight g-coherent (resp., logical) consequences of $K B_{1}$ and $K B_{2}$ are given by:

$$
\begin{aligned}
& K B_{1} \sim_{\text {tight }}(\text { mobile } \mid \text { bird })[0,1] \text { and } K B_{2} \sim_{\text {tight }}(\neg \text { fly|penguin })[1,1], \\
& K B_{1} \models_{\text {tight }}(\text { mobile|bird })[1,1] \text { and } K B_{2} \models_{\text {tight }}(\neg \text { fly|penguin })[1,0] .
\end{aligned}
$$

\section{Relationship to default reasoning in System $P$}

In this section, we explore the relationship between probabilistic reasoning under coherence and default reasoning in System $P$. We show that consistency and entailment in System $P$ are special cases of g-coherence and g-coherent entailment, respectively. That is, probabilistic logic under coherence gives a new probabilistic semantics for System $P$, which is neither based on infinitesimal probabilities [1,34] nor on atomic bound [39] (or also big-stepped [4]) probabilities.

\subsection{Default reasoning in System $P$}

We briefly recall the notions of consistency and entailment in System $P$ [25], which we define in terms of world rankings (cf. especially $[18,17]$ for the equivalence between entailment in System $P$ and entailment under world rankings).

We first define conditional knowledge bases, which consist of logical constraints $\psi \Leftarrow \phi$ and conditional rules (or defaults) $\psi \leftarrow \phi$, which informally read as "generally, if $\phi$ then $\psi$ ". More formally, a conditional rule (or default) is an expression of the form $\psi \leftarrow \phi$, where $\phi$ and $\psi$ are events. A conditional knowledge base $K B=(L, D)$ consists of a finite set of logical constraints $L$ and a finite set of defaults $D$.

A world $I$ satisfies a default $\psi \leftarrow \phi$, or $I$ is a model of $\psi \leftarrow \phi$, denoted $I \models \psi \leftarrow \phi$, iff $I \models \psi \Leftarrow \phi$. I verifies $\psi \leftarrow \phi$ iff $I \models \phi \wedge \psi$. I falsifies $\psi \leftarrow \phi$ iff $I \models \phi \wedge \neg \psi$ (that is, $I \not \models \psi \leftarrow \phi$ ). I satisfies a set of events and defaults $K$, or $I$ is a model of $K$, denoted $I \models K$, iff $I$ satisfies every member of $K$. We say $K$ is satisfiable iff a model of $K$ exists. An event $\phi$ is a logical consequence of $K$, denoted $K \models \phi$, iff every model of $K$ is also a model of $\phi$. A set of defaults $D$ tolerates a default $d$ under a set of logical constraints $L$ iff $D \cup L$ has a model that verifies $d$. A set of defaults $D$ is under $L$ in conflict with a default $\psi \leftarrow \phi$ iff all models of $D \cup L \cup\{\phi\}$ satisfy $\neg \psi$.

A world ranking $\kappa$ is a mapping $\kappa: \mathcal{I}_{\Phi} \rightarrow\{0,1, \ldots\} \cup\{\infty\}$ such that $\kappa(I)=0$ for at least one world $I$. It is extended to all events $\phi$ as follows. If $\phi$ is satisfiable, then $\kappa(\phi)=\min \left\{\kappa(I) \mid I \in \mathcal{I}_{\Phi}, I \models \phi\right\}$; 
otherwise, $\kappa(\phi)=\infty$. A world ranking $\kappa$ is admissible with a conditional knowledge base $(L, D)$ iff $\kappa(\neg \phi)=\infty$ for all $\phi \in L$, and $\kappa(\phi)<\infty$ and $\kappa(\phi \wedge \psi)<\kappa(\phi \wedge \neg \psi)$ for all defaults $\psi \leftarrow \phi \in D$.

We are now ready to define the notions of consistency and entailment in System $P$. A conditional knowledge base $K B$ is $p$-consistent iff there exists a world ranking that is admissible with $K B$. It is $p$ inconsistent iff no such a world ranking exists. A $p$-consistent conditional knowledge base $K B$-entails a default $\psi \leftarrow \phi$ iff either $\kappa(\phi)=\infty$ or $\kappa(\phi \wedge \psi)<\kappa(\phi \wedge \neg \psi)$ for all world rankings $\kappa$ admissible with $K B$.

Example 4.1 The logical knowledge "all penguins are birds" and the logical default knowledge "generally, birds have legs", "generally, birds fly", and "generally, penguins do not fly" can be expressed by the following conditional knowledge base:

$$
K B=(\{\text { bird } \Leftarrow \text { penguin }\},\{\text { legs } \leftarrow \text { bird, fly } \leftarrow \text { bird, } \neg \text { fly } \leftarrow \text { penguin }\}) .
$$

Observe that $K B$ is $p$-consistent, and that $K B p$-entails legs $\leftarrow$ bird, fly $\leftarrow$ bird, and $\neg$ fly $\leftarrow$ penguin, but neither legs $\leftarrow$ penguin nor $\neg$ legs $\leftarrow$ penguin.

\subsection{G-coherence and $p$-consistency}

We now show that g-coherence is a generalization of $p$-consistency. Recall first the following characterization of $p$-consistency by Goldszmidt and Pearl [23], which corresponds to the characterization of g-coherence given in Theorem 3.3.

Theorem 4.2 (Goldszmidt, Pearl [23]) A conditional knowledge base $(L, D)$ is $p$-consistent iff there exists an ordered partition $\left(D_{0}, \ldots, D_{k}\right)$ of $D$ such that either

(a) every $D_{i}, 0 \leq i \leq k$, is the set of all $d \in \bigcup_{j=i}^{k} D_{j}$ tolerated under $L$ by $\bigcup_{j=i}^{k} D_{j}$, or

(b) for every $i, 0 \leq i \leq k$, each $d \in D_{i}$ is tolerated under $L$ by $\bigcup_{j=i}^{k} D_{j}$.

The following well-known result shows that $p$-consistency is equivalent to the existence of admissible default rankings. A default ranking $\sigma$ on $K B=(L, D)$ maps each $d \in D$ to a nonnegative integer. It is admissible with $K B$ iff each $D^{\prime} \subseteq D$ that is under $L$ in conflict with some $d \in D$ contains a default $d^{\prime}$ such that $\sigma\left(d^{\prime}\right)<\sigma(d)$.

Theorem 4.3 (Geffner [17]) A conditional knowledge base $K B$ is $p$-consistent iff there exists a default ranking on $K B$ that is admissible with $K B$.

A similar result holds for the notion of g-coherence, which follows from Theorem 3.3 (b), and which is subsequently formulated using the following concepts. A ranking $\sigma$ on $K B=(L, P)$ maps each element of $P$ to a nonnegative integer. It is admissible with $K B$ iff each $P^{\prime} \subseteq P$ that is under $L$ in conflict with some $F \in P$ contains a conditional constraint $F^{\prime}$ such that $\sigma\left(F^{\prime}\right)<\sigma(F)$.

Theorem 4.4 A probabilistic knowledge base $K B=(L, P)$ is g-coherent iff there exists a ranking on KB that is admissible with $K B$.

The next theorem shows the important result that g-coherence is a generalization of $p$-consistency. It follows from Theorems 3.3 (b) and 4.2 (b), using the result that probabilistic toleration for $(\psi \mid \phi)[1,1]$ coincides with classical toleration for $\psi \leftarrow \phi$. 
Theorem 4.5 Let $K B=\left(L,\left\{\left(\psi_{1} \mid \phi_{1}\right)[1,1], \ldots,\left(\psi_{n} \mid \phi_{n}\right)[1,1]\right\}\right)$ be a probabilistic knowledge base. Then, $K B$ is $g$-coherent iff the conditional knowledge base $K B^{\prime}=\left(L,\left\{\psi_{1} \leftarrow \phi_{1}, \ldots, \psi_{n} \leftarrow \phi_{n}\right\}\right)$ is p-consistent.

\subsection{G-coherent entailment and $p$-entailment}

We now show that g-coherent entailment is a generalization of $p$-entailment. We recall the following result, which is essentially due to Adams [1], who formulated it for the notions of $\varepsilon$-consistency and $\varepsilon$-entailment, and the case $L=\emptyset$.

Theorem 4.6 (Adams [1]) A p-consistent conditional knowledge base $K B=(L, D)$ p-entails a default $\beta \leftarrow \alpha$ iff $(L, D \cup\{\neg \beta \leftarrow \alpha\})$ is p-inconsistent.

The following theorem shows that a similar result holds for g-coherent consequence. It is an immediate implication of the definition of g-coherent entailment.

Theorem 4.7 Let $K B=(L, P)$ be a g-coherent probabilistic knowledge base, and let $(\beta \mid \alpha)[l, u]$ be a conditional constraint. Then, $K B \sim(\beta \mid \alpha)[l, u]$ iff $(L, P \cup\{(\beta \mid \alpha)[p, p]\})$ is not g-coherent for all $p \in[0, l) \cup(u, 1]$.

The next theorem for tight g-coherent consequence completes the picture. This result follows immediately from the proof of Theorem 3.4.

Theorem 4.8 Let $K B=(L, P)$ be a g-coherent probabilistic knowledge base, and let $(\beta \mid \alpha)[l, u]$ be a conditional constraint. Then, $K B \sim_{\text {tight }}(\beta \mid \alpha)[l, u]$ iff

(i) $(L, P \cup\{(\beta \mid \alpha)[p, p]\})$ is not g-coherent for all $p \in[0, l) \cup(u, 1]$, and

(ii) $(L, P \cup\{(\beta \mid \alpha)[p, p]\})$ is $g$-coherent for all $p \in[l, u]$.

The following theorem finally shows that g-coherent entailment is a generalization of $p$-entailment. This result follows from Theorems 3.2 and 4.5-4.7.

Theorem 4.9 Let $K B=\left(L,\left\{\left(\psi_{1} \mid \phi_{1}\right)[1,1], \ldots,\left(\psi_{n} \mid \phi_{n}\right)[1,1]\right\}\right)$ be a g-coherent probabilistic knowledge base. Then, $K B \sim(\beta \mid \alpha)[1,1]$ iff the conditional knowledge base $\left(L,\left\{\psi_{1} \leftarrow \phi_{1}, \ldots, \psi_{n} \leftarrow \phi_{n}\right\}\right) p$-entails $\beta \leftarrow \alpha$.

\section{Relationship to default reasoning with conditional objects}

We now relate probabilistic reasoning under coherence to default reasoning with conditional objects, which goes back to Dubois and Prade [13, 3].

We associate with each set of defaults $D=\left\{\psi_{1} \leftarrow \phi_{1}, \ldots, \psi_{n} \leftarrow \phi_{n}\right\}$, the set of conditional events $\mathcal{C}_{D}=\left\{\psi_{1}\left|\phi_{1}, \ldots, \psi_{n}\right| \phi_{n}\right\}$. Given a nonempty set of conditional events $\mathcal{C}=\left\{\psi_{1}\left|\phi_{1}, \ldots, \psi_{n}\right| \phi_{n}\right\}$, the quasiconjunction of $\mathcal{C}$, denoted $Q C(\mathcal{C})$, is defined as the conditional event $\left(\psi_{1} \Leftarrow \phi_{1}\right) \wedge \cdots \wedge\left(\psi_{n} \Leftarrow \phi_{n}\right) \mid$ $\phi_{1} \vee \cdots \vee \phi_{n}$. The quasi-conjunction of $\emptyset$ is defined by $Q C(\emptyset)=\top \mid \perp$. Note that the notion of quasiconjunction goes back to Adams [1].

The notions of co-consistency and co-entailment are defined as follows. A conditional knowledge base $K B=(L, D)$ is co-consistent iff for every nonempty $D^{\prime} \subseteq D$ there exists a model $I$ of $L$ such that $I\left(Q C\left(\mathcal{C}_{D^{\prime}}\right)\right)=$ true. We assume the total order false $<$ indeterminate $<$ true. We say $K B=(L, D)$ 
co-entails a default $\beta \leftarrow \alpha$ iff $(\star)$ some $D^{\prime} \subseteq D$ exists such that $I\left(Q C\left(\mathcal{C}_{D^{\prime}}\right)\right) \leq I(\beta \mid \alpha)$ for all models $I$ of $L$.

The notions of co-consistency and co-entailment coincide with the notions of $p$-consistency and $p$ entailment, respectively [13,3]. We now show that our results in Sections 3 and 4 are naturally related to default reasoning with conditional objects. First, it is easy to verify that the following counterpart of Theorem 3.2 for $p$-consistency formulates the above notion of co-consistency. Note that the notion of satisfiability used in this theorem is defined as in Section 4.1.

Theorem 5.1 A conditional knowledge base $K B=(L, D)$ is p-consistent iff $L \cup D^{\prime} \cup\left\{\phi_{1} \vee \cdots \vee \phi_{n}\right\}$ is satisfiable for every nonempty $D^{\prime}=\left\{\psi_{1} \leftarrow \phi_{1}, \ldots, \psi_{n} \leftarrow \phi_{n}\right\} \subseteq D$.

For conditional knowledge bases $K B=(L, D)$ and events $\alpha$ with $L \not \neq \neg \alpha$, let $D_{\alpha}(K B)$ be the set of all $D^{\prime}=\left\{\psi_{1} \leftarrow \phi_{1}, \ldots, \psi_{n} \leftarrow \phi_{n}\right\} \subseteq D$ such that $L \cup D^{\prime} \cup\left\{\phi_{1} \vee \cdots \vee \phi_{n} \vee \alpha\right\} \models \alpha$ (where the notion of logical consequence " $="$ " is defined as in Section 4.1). For $K B=(L, D)$ and $\alpha$ with $L \models \neg \alpha$, let $D_{\alpha}(K B)=\{\emptyset\}$.

The following lemma shows that $D_{\alpha}(K B)$ corresponds to $P_{\alpha}\left(K B^{\prime}\right)$ from Section 3.3, where $K B^{\prime}$ is the probabilistic counterpart to $K B$.

Lemma 5.2 Let $K B=(L, D)$ be a conditional knowledge base, and let $\alpha$ be an event. Let $K B^{\prime}=(L, P)$ be defined by $P=\{(\psi \mid \phi)[1,1] \mid \psi \leftarrow \phi \in D\}$. Then, $\left\{\psi_{1} \leftarrow \phi_{1}, \ldots, \psi_{n} \leftarrow \phi_{n}\right\} \in D_{\alpha}(K B)$ iff $\left\{\left(\psi_{1} \mid \phi_{1}\right)[1,1]\right.$, $\left.\ldots,\left(\psi_{n} \mid \phi_{n}\right)[1,1]\right\} \in P_{\alpha}\left(K B^{\prime}\right)$.

Observe now that condition $(\star)$ above is equivalent to $\left(\star^{\prime}\right)$ the existence of some $D^{\prime}=\left\{\psi_{1} \leftarrow \phi_{1}, \ldots\right.$, $\left.\psi_{n} \leftarrow \phi_{n}\right\} \subseteq D$ such that $L \cup D^{\prime} \cup\left\{\phi_{1} \vee \cdots \vee \phi_{n} \vee \alpha\right\} \models \alpha$ and $L \cup D^{\prime} \models \beta \Leftarrow \alpha$. Thus, the following counterpart of Theorem 3.4 for $p$-entailment formulates the above notion of co-entailment.

Theorem 5.3 Let $K B=(L, D)$ be a $p$-consistent conditional knowledge base. Then, $K B$-entails the default $\beta \leftarrow \alpha$ iff $L \cup D^{\prime} \models \beta \Leftarrow \alpha$ for some $D^{\prime} \in D_{\alpha}(K B)$.

Crucially, we can now also formulate counterparts to Lemma 3.5 and Theorems 3.6 and 3.7. The following result shows that $D_{\alpha}(K B)$ contains a unique greatest element.

Lemma 5.4 Let $K B=(L, D)$ be a p-consistent conditional knowledge base, and let $\alpha$ be an event. Then, $D_{\alpha}(K B)$ contains a unique greatest element.

The next theorem shows that $p$-entailment from $K B$ coincides with logical entailment from the greatest element in $D_{\alpha}(K B)$. That is, denoting by $D^{\star}$ the greatest element in $D_{\alpha}(K B)$, we can replace condition $(\star)$ above by $\left(\star^{\prime \prime}\right) I\left(Q C\left(\mathcal{C}_{D^{\star}}\right)\right) \leq I(\beta \mid \alpha)$ for all models $I$ of $L$, or simply by $\left(\star^{\prime \prime \prime}\right) L \cup D^{\star} \models \beta \Leftarrow \alpha$.

Theorem 5.5 Let $K B=(L, D)$ be a p-consistent conditional knowledge base, and let $\beta \leftarrow \alpha$ be a default. Let $D^{\star}$ denote the unique greatest element in $D_{\alpha}(K B)$. Then, KB p-entails $\beta \leftarrow \alpha$ iff $L \cup D^{\star}=\beta \Leftarrow \alpha$.

The following theorem shows how $D^{\star}$ can be characterized.

Theorem 5.6 Let $K B=(L, D)$ be a p-consistent conditional knowledge base, and let $\alpha$ be an event. Let $D^{\star} \subseteq D$, and let $\left(D_{0}, \ldots, D_{k}\right)$ be an ordered partition of $D \backslash D^{\star}$ such that the following two conditions (i) and (ii) hold: 
(i) Every $D_{i}, 0 \leq i \leq k$, is the set of all elements in $D_{i} \cup \cdots \cup D_{k} \cup D^{\star}$ that are tolerated under $L \cup$ $\{\perp \Leftarrow \alpha\}$ by $D_{i} \cup \cdots \cup D_{k} \cup D^{\star}$.

(ii) No member of $D^{\star}$ is tolerated under $L \cup\{\perp \Leftarrow \alpha\}$ by $D^{\star}$.

Then, $D^{\star}$ is the greatest element in $D_{\alpha}(K B)$.

Observe especially that Theorems 5.5 and 5.6 provide a new algorithm for deciding whether $K B=$ $(L, D) p$-entails $\beta \leftarrow \alpha$. We first compute $D^{\star}$ by a $p$-consistency check, and then we decide whether $L \cup D^{\star}$ logically entails $\beta \Leftarrow \alpha$.

\section{Conclusion and outlook}

We explored how probabilistic reasoning under coherence is related to model-theoretic probabilistic reasoning and to default reasoning in System $P$.

As for the relationship between probabilistic logic under coherence and model-theoretic probabilistic logic, we showed that g-coherence and g-coherent entailment can be reduced to the existence of satisfying probabilistic interpretations and to logical entailment, respectively, using concepts from default reasoning. We observed especially that g-coherent entailment coincides with logical entailment from a smaller knowledge base, and that logical entailment has the property of inheritance of logical knowledge, while g-coherent entailment does not. Furthermore, g-coherence is stronger than satisfiability and weaker than strong satisfiability, while g-coherent entailment is weaker than logical entailment.

The above semantic results also paved the way for a precise complexity characterization and new algorithms for the problems of deciding g-coherence and of computing tight g-coherent intervals. Note especially that the new algorithms for deciding g-coherence and for computing tight g-coherent intervals are based on a reduction to standard reasoning tasks in model-theoretic probabilistic logic, and thus efficient techniques for model-theoretic probabilistic reasoning can immediately be applied for probabilistic reasoning under coherence (for example, column generation techniques). These new results on complexity and algorithms for probabilistic reasoning under coherence have been presented in a companion paper [6].

Note that a companion paper [30] continues this line of research on the relationship between coherencebased and model-theoretic probabilistic reasoning. In the spirit of [31], it introduces probabilistic generalizations of Pearl's entailment in System $Z$ [35] and Lehmann's lexicographic entailment [26], which lie between g-coherent entailment and logical entailment.

As for the relationship between probabilistic reasoning under coherence and default reasoning in System $P$, we showed that g-coherence and g-coherent entailment are generalizations of consistency and entailment in System $P$, respectively. This result gives a clear picture of the main properties of probabilistic reasoning under coherence, and the difference to model-theoretic probabilistic reasoning, which generalizes classical reasoning in propositional logic, and not default reasoning in System $P$.

These results also give important new insight into default reasoning in System $P$. First and foremost, they show that probabilistic reasoning under coherence gives a new probabilistic semantics for System $P$, which is neither based on infinitesimal probabilities [1,34] nor on atomic bound [39] (or also big-stepped [4]) probabilities. Note that an intuitive idea on the structure of this new probabilistic semantics for System $P$ is given by the fact that probabilistic reasoning under coherence can be defined in terms of rankings on probabilistic interpretations [30]. Second, these results provide new insight into default reasoning with conditional objects, by showing that the definitions of co-consistency and co-entailment are actually classical counterparts of the definitions of g-coherence and g-coherent entailment, respectively. Third, they 
provide a new algorithm for default reasoning in System $P$, which differs from the classical one based on Theorem 4.6.

An interesting topic of future research is to explore how other notions of coherence are related to modeltheoretic probabilistic logic and to default reasoning.

\section{Appendix A: Proofs for Section 3}

Proof of Theorem 3.2. Let $P_{n}=\left\{\left(\psi_{1} \mid \phi_{1}\right)\left[l_{1}, u_{1}\right], \ldots,\left(\psi_{n} \mid \phi_{n}\right)\left[l_{n}, u_{n}\right]\right\} \subseteq P$ with $n \geq 1$. Define the imprecise probability assessment $(L, A)$ on $\mathcal{C}_{n}=\left\{\psi_{1}\left|\phi_{1}, \ldots, \psi_{n}\right| \phi_{n}\right\}$ by $A\left(\psi_{i} \mid \phi_{i}\right)=\left[l_{i}, u_{i}\right]$ for all $i \in\{1, \ldots, n\}$. By Theorem 2.1, it is sufficient to show that the system of linear constraints (1) is solvable iff $L \cup P_{n}$ has a model $\operatorname{Pr}$ with $\operatorname{Pr}\left(\phi_{1} \vee \cdots \vee \phi_{n}\right)>0$. Observe first that $R$ defines a partition $\mathcal{S}=\left\{S_{r} \mid r \in R\right\}$ of the set of all $I \in \mathcal{I}_{\Phi}$ with $I \models L \cup\left\{\phi_{1} \vee \cdots \vee \phi_{n}\right\}$, where $S_{r}=\left\{I \in \mathcal{I}_{\Phi} \mid I \models L \cup\left\{\phi_{1} \vee \cdots \vee \phi_{n}\right\}\right.$, $\left.I \models r\left(\psi_{1} \mid \phi_{1}\right) \wedge \cdots \wedge r\left(\psi_{n} \mid \phi_{n}\right)\right\}$ for all $r \in R$.

$(\Rightarrow)$ Suppose that (1) has a solution $\lambda_{r}(r \in R)$. For each $r \in R$, denote by $I_{r}$ a world in $\mathcal{I}_{\Phi}$ that satisfies $L$ and $r\left(\psi_{1} \mid \phi_{1}\right) \wedge \cdots \wedge r\left(\psi_{n} \mid \phi_{n}\right)$. Let $\operatorname{Pr}$ be defined by $\operatorname{Pr}\left(I_{r}\right)=\lambda_{r}$ for all $r \in R$ and by $\operatorname{Pr}(I)=0$ for all other $I \in \mathcal{I}_{\Phi}$. Clearly, $\operatorname{Pr}$ is a probabilistic interpretation with $\operatorname{Pr}\left(\phi_{1} \vee \cdots \vee \phi_{n}\right)=1>0$. Moreover, $\operatorname{Pr}$ satisfies $L$, and $\operatorname{Pr}$ satisfies the following for all $i \in\{1, \ldots, n\}$ :

$$
\begin{aligned}
& 1 \cdot \operatorname{Pr}\left(\psi_{i} \wedge \phi_{i}\right)+0 \cdot \operatorname{Pr}\left(\neg \psi_{i} \wedge \phi_{i}\right)+l_{i} \cdot \operatorname{Pr}\left(\neg \phi_{i}\right) \geq l_{i}, \\
& 1 \cdot \operatorname{Pr}\left(\psi_{i} \wedge \phi_{i}\right)+0 \cdot \operatorname{Pr}\left(\neg \psi_{i} \wedge \phi_{i}\right)+u_{i} \cdot \operatorname{Pr}\left(\neg \phi_{i}\right) \leq u_{i} .
\end{aligned}
$$

The latter is equivalent to $l_{i} \cdot \operatorname{Pr}\left(\phi_{i}\right) \leq \operatorname{Pr}\left(\psi_{i} \wedge \phi_{i}\right) \leq u_{i} \cdot \operatorname{Pr}\left(\phi_{i}\right)$, and thus $\operatorname{Pr} \models\left(\psi_{i} \mid \phi_{i}\right)\left[l_{i}, u_{i}\right]$ for all $i \in\{1, \ldots, n\}$. In summary, $\operatorname{Pr}$ is a model of $L \cup P_{n}$ such that $\operatorname{Pr}\left(\phi_{1} \vee \cdots \vee \phi_{n}\right)>0$.

$(\Leftarrow)$ Conversely, let $\operatorname{Pr}$ be a model of $L \cup P_{n}$ with $\operatorname{Pr}\left(\phi_{1} \vee \cdots \vee \phi_{n}\right)>0$. Define now the probabilistic interpretation $\operatorname{Pr}^{\prime}$ by $\operatorname{Pr}^{\prime}(I)=0$ for all $I \in \mathcal{I}_{\Phi}$ with $I \not \neq \phi_{1} \vee \cdots \vee \phi_{n}$ and by $\operatorname{Pr}^{\prime}(I)=$ $\operatorname{Pr}(I) / \operatorname{Pr}\left(\phi_{1} \vee \cdots \vee \phi_{n}\right)$ for all other $I \in \mathcal{I}_{\Phi}$. Clearly, $\operatorname{Pr}^{\prime}$ is a model of $L \cup P_{n}$ with $\operatorname{Pr}^{\prime}\left(\phi_{1} \vee \cdots \vee \phi_{n}\right)=$ 1. In particular, for all $i \in\{1, \ldots, n\}$, it holds $\operatorname{Pr}^{\prime} \models\left(\psi_{i} \mid \phi_{i}\right)\left[l_{i}, u_{i}\right]$, or equivalently $l_{i} \cdot \operatorname{Pr}^{\prime}\left(\phi_{i}\right) \leq$ $\operatorname{Pr}^{\prime}\left(\psi_{i} \wedge \phi_{i}\right) \leq u_{i} \cdot \operatorname{Pr}^{\prime}\left(\phi_{i}\right)$, which in turn is equivalent to:

$$
\begin{aligned}
& 1 \cdot \operatorname{Pr}^{\prime}\left(\psi_{i} \wedge \phi_{i}\right)+0 \cdot \operatorname{Pr}^{\prime}\left(\neg \psi_{i} \wedge \phi_{i}\right)+l_{i} \cdot \operatorname{Pr}^{\prime}\left(\neg \phi_{i}\right) \geq l_{i}, \\
& 1 \cdot \operatorname{Pr}^{\prime}\left(\psi_{i} \wedge \phi_{i}\right)+0 \cdot \operatorname{Pr}^{\prime}\left(\neg \psi_{i} \wedge \phi_{i}\right)+u_{i} \cdot \operatorname{Pr}^{\prime}\left(\neg \phi_{i}\right) \leq u_{i} .
\end{aligned}
$$

For all $r \in R$, define finally $\lambda_{r}=\operatorname{Pr}^{\prime}\left(r\left(\psi_{1} \mid \phi_{1}\right) \wedge \cdots \wedge r\left(\psi_{n} \mid \phi_{n}\right)\right)$. It is now easy to verify that $\lambda_{r}(r \in R)$ is a solution of (1).

Proof of Theorem 3.3. $(\Rightarrow)$ Suppose that $K B$ is g-coherent. By Theorem 3.2, for every nonempty $P^{\prime}=\left\{\left(\psi_{1} \mid \phi_{1}\right)\left[l_{1}, u_{1}\right], \ldots,\left(\psi_{n} \mid \phi_{n}\right)\left[l_{n}, u_{n}\right]\right\} \subseteq P$, there exists a model $\operatorname{Pr}$ of $L \cup P^{\prime}$ such that $\operatorname{Pr}\left(\phi_{1} \vee\right.$ $\left.\cdots \vee \phi_{n}\right)>0$, and thus $\operatorname{Pr}\left(\phi_{j}\right)>0$ for some $j \in\{1, \ldots, n\}$. That is, every nonempty $P^{\prime} \subseteq P$ contains a conditional constraint that is tolerated under $L$ by $P^{\prime}$. Then $P=\bigcup_{j=0}^{k} P_{j}$, where

$$
\begin{aligned}
& P_{0}=\{F \in P \mid F \text { is tolerated under } L \text { by } P\} \\
& P_{i}=\left\{F \in P_{i}^{*} \mid F \text { is tolerated under } L \text { by } P_{i}^{*}\right\}, \\
& \text { where } P_{i}^{*}=P \backslash \bigcup_{j=0}^{i-1} P_{j}=\bigcup_{j=i}^{k} P_{j}, \text { for all } i \in\{1, \ldots, k\} .
\end{aligned}
$$

In particular, $P_{k}=P \backslash \bigcup_{j=0}^{k-1} P_{j}$ is such that each $F \in P_{k}$ is tolerated under $L$ by $P_{k}$. This shows that (a) and (b) hold. 
$(\Leftarrow)$ Conversely, as (a) implies (b), we can assume that (b) holds. That is, suppose that, for every $i \in\{0, \ldots, k\}$, each member of $P_{i}$ is tolerated under $L$ by $P_{i}^{*}=P_{i} \cup \cdots \cup P_{k}$. That is, for each $(\psi \mid \phi)[l, u] \in P_{i}$, there is a model $\operatorname{Pr}$ of $L \cup P_{i}^{*}$ with $\operatorname{Pr}(\phi)>0$. Thus, for each $P^{\prime}=\left\{\left(\psi_{1} \mid \phi_{1}\right)\left[l_{1}, u_{1}\right], \ldots\right.$, $\left.\left(\psi_{n} \mid \phi_{n}\right)\left[l_{n}, u_{n}\right]\right\} \subseteq P$, denoting by $i$ the minimum integer with $P^{\prime} \cap P_{i} \neq \emptyset$, there is some $\left(\psi_{j} \mid \phi_{j}\right)\left[l_{j}, u_{j}\right] \in$ $P^{\prime} \cap P_{i}$ tolerated under $L$ by $P_{i}^{*}$, and then $\left(\psi_{j} \mid \phi_{j}\right)\left[l_{j}, u_{j}\right]$ is also tolerated under $L$ by $P^{\prime}$. That is, $L \cup P^{\prime}$ has a model $\operatorname{Pr}$ with $\operatorname{Pr}\left(\phi_{j}\right)>0$ and thus $\operatorname{Pr}\left(\phi_{1} \vee \cdots \vee \phi_{n}\right)>0$. Then, by Theorem 3.2, it follows that $P$ is g-coherent.

Proof of Theorem 3.4. Suppose that $[l, u]$ is given by $K B \sim_{\text {tight }}(\beta \mid \alpha)[l, u]$ and that $[r, s]$ is the intersection of all $\left[l^{\prime}, u^{\prime}\right]$ such that $L \cup P^{\prime} \models_{\text {tight }}(\beta \mid \alpha)\left[l^{\prime}, u^{\prime}\right]$ for some $P^{\prime} \in P_{\alpha}(K B)$. We now show that $[l, u]=[r, s]$.

We first prove that $[l, u] \subseteq[r, s]$. Recall that $l$ (resp., $u$ ) is the infimum (resp., supremum) of $p$ subject to all g-coherent $K B^{\prime}=\left(L, P^{\prime}\right)=(L, P \cup\{(\beta \mid \alpha)[p, p]\})$. By Theorem 3.2,KB' is g-coherent iff for every nonempty $P_{n}=\left\{\left(\psi_{1} \mid \phi_{1}\right)\left[l_{1}, u_{1}\right], \ldots,\left(\psi_{n} \mid \phi_{n}\right)\left[l_{n}, u_{n}\right]\right\} \subseteq P^{\prime}$ there exists a model $\operatorname{Pr}$ of $L \cup P_{n}$ with $\operatorname{Pr}\left(\phi_{1} \vee \cdots \vee \phi_{n}\right)>0$. In particular, g-coherence of $K B^{\prime}$ implies that for each $P^{\prime \prime} \in P_{\alpha}(K B)$, there is a model $\operatorname{Pr}$ of $L \cup P^{\prime \prime} \cup\{(\beta \mid \alpha)[p, p]\}=L \cup\left\{\left(\psi_{1} \mid \phi_{1}\right)\left[l_{1}, u_{1}\right], \ldots,\left(\psi_{n} \mid \phi_{n}\right)\left[l_{n}, u_{n}\right],(\beta \mid \alpha)[p, p]\right\}$ with $\operatorname{Pr}\left(\phi_{1} \vee \cdots \vee \phi_{n} \vee \alpha\right)>0$, or equivalently $\operatorname{Pr}(\alpha)>0$. Recall now that $r$ (resp., $s$ ) is given by the infimum (resp., supremum) of $\operatorname{Pr}(\beta \mid \alpha)$ subject to $\operatorname{Pr} \models L \cup P^{\prime \prime}, \operatorname{Pr}(\alpha)>0$, and $P^{\prime \prime} \in P_{\alpha}(K B)$. It thus follows $p \in[r, s]$. That is, $[l, u] \subseteq[r, s]$.

We next show that for every element $p \in[r, s]$, it holds that $K B^{\prime}=\left(L, P^{\prime}\right)=(L, P \cup\{(\beta \mid \alpha)[p, p]\})$ is g-coherent. Towards a contradiction, suppose not. By Theorem 3.3, there exists an ordered partition $\left(P_{0}, \ldots, P_{k}\right)$ of $P^{\prime}$ such that (i) each $P_{i}$ with $i \in\{0, \ldots, k-1\}$ is the set of all elements in $P_{i} \cup \cdots \cup$ $P_{k}$ that are tolerated under $L$ by $P_{i} \cup \cdots \cup P_{k}$, and (ii) no member of $P_{k}$ is tolerated under $L$ by $P_{k}$. Assume now that $(\beta \mid \alpha)[p, p] \notin P_{k}$. By Theorem 3.2, the g-coherence of $K B$ implies that $L \cup P_{k}=L \cup$ $\left\{\left(\psi_{1} \mid \phi_{1}\right)\left[l_{1}, u_{1}\right], \ldots,\left(\psi_{n} \mid \phi_{n}\right)\left[l_{n}, u_{n}\right]\right\}$ has a model $\operatorname{Pr}$ with $\operatorname{Pr}\left(\phi_{1} \vee \cdots \vee \phi_{n}\right)>0$, and thus $\operatorname{Pr}\left(\phi_{j}\right)>0$ for some $j \in\{1, \ldots, n\}$. That is, $\left(\psi_{j} \mid \phi_{j}\right)\left[l_{j}, u_{j}\right] \in P_{k}$ is tolerated under $L$ by $P_{k}$. But this contradicts (ii). It thus follows $(\beta \mid \alpha)[p, p] \in P_{k}$. Assume that $L \cup P_{k}^{\prime}=L \cup P_{k}-\{(\beta \mid \alpha)[p, p]\}=L \cup\left\{\left(\psi_{1}^{\prime} \mid \phi_{1}^{\prime}\right)\left[l_{1}^{\prime}, u_{1}^{\prime}\right], \ldots\right.$, $\left.\left(\psi_{n}^{\prime} \mid \phi_{n}^{\prime}\right)\left[l_{n}^{\prime}, u_{n}^{\prime}\right]\right\}$ has a model $\operatorname{Pr}$ with $\operatorname{Pr}(\alpha)=0$ and $\operatorname{Pr}\left(\phi_{1}^{\prime} \vee \cdots \vee \phi_{n}^{\prime}\right)>0$, and thus $\operatorname{Pr}\left(\phi_{j}^{\prime}\right)>0$ for some $j \in\{1, \ldots, n\}$. Trivially, $\operatorname{Pr}$ also satisfies $(\beta \mid \alpha)[p, p]$. Hence, $\left(\psi_{j}^{\prime} \mid \phi_{j}^{\prime}\right)\left[l_{j}^{\prime}, u_{j}^{\prime}\right] \in P_{k}$ is tolerated under $L$ by $P_{k}$. But this contradicts again (ii). Thus, every model $\operatorname{Pr}$ of $L \cup P_{k}^{\prime}$ with $\operatorname{Pr}\left(\phi_{1}^{\prime} \vee \cdots \vee \phi_{n}^{\prime} \vee \alpha\right)>0$ satisfies $\operatorname{Pr}(\alpha)>0$. Hence, $P_{k}^{\prime} \in P_{\alpha}(K B)$. Since $p \in[r, s]$, there exists a model $\operatorname{Pr}$ of $L \cup P_{k}^{\prime}$ such that $\operatorname{Pr}=(\beta \mid \alpha)[p, p]$ and $\operatorname{Pr}(\alpha)>0$. That is, $(\beta \mid \alpha)[p, p] \in P_{k}$ is tolerated under $L$ by $P_{k}$. But this contradicts again (ii). It thus follows that $K B^{\prime}$ is g-coherent.

Proof of Lemma 3.5. Obviously, $P_{\alpha}(K B)$ is not empty, as it contains $\emptyset$. Towards a contradiction, suppose now that $P_{m}=\left\{\left(\psi_{1} \mid \phi_{1}\right)\left[l_{1}, u_{1}\right], \ldots,\left(\psi_{m} \mid \phi_{m}\right)\left[l_{m}, u_{m}\right]\right\}$ and $P_{n}=\left\{\left(\psi_{1}^{\prime} \mid \phi_{1}^{\prime}\right)\left[l_{1}^{\prime}, u_{1}^{\prime}\right], \ldots,\left(\psi_{n}^{\prime} \mid \phi_{n}^{\prime}\right)\left[l_{n}^{\prime}, u_{n}^{\prime}\right]\right\}$ are two distinct maximal elements in $P_{\alpha}(K B)$. That is, every model $\operatorname{Pr}$ of $L \cup P_{m}$ (resp., $L \cup P_{n}$ ) with $\operatorname{Pr}\left(\phi_{1} \vee \cdots \vee \phi_{m} \vee \alpha\right)>0$ (resp., $\operatorname{Pr}\left(\phi_{1}^{\prime} \vee \cdots \vee \phi_{n}^{\prime} \vee \alpha\right)>0$ ) satisfies $\operatorname{Pr}(\alpha)>0$. Consider now any model $\operatorname{Pr}$ of $L \cup P_{m} \cup P_{n}$ with $\operatorname{Pr}\left(\phi_{1} \vee \cdots \vee \phi_{m} \vee \phi_{1}^{\prime} \vee \cdots \vee \phi_{n}^{\prime} \vee \alpha\right)>0$. Clearly, $\operatorname{Pr}$ is a model of both $L \cup P_{m}$ and $L \cup P_{n}$ such that either $\operatorname{Pr}\left(\phi_{1} \vee \cdots \vee \phi_{m} \vee \alpha\right)>0$ or $\operatorname{Pr}\left(\phi_{1}^{\prime} \vee \cdots \vee \phi_{n}^{\prime} \vee \alpha\right)>0$. It thus follows $\operatorname{Pr}(\alpha)>0$. Hence, $P_{m} \cup P_{n}$ belongs to $P_{\alpha}(K B)$. But this contradicts $P_{m}$ and $P_{n}$ being two distinct maximal elements in $P_{\alpha}(K B)$. It thus follows that $P_{\alpha}(K B)$ contains a unique greatest element.

Proof of Theorem 3.6. (a) Immediate by (b).

(b) Immediate by Theorem 3.4 and Lemma 3.5, since the conditions $P^{\prime} \subseteq P^{\prime \prime}, L \cup P^{\prime} \models_{\text {tight }}(\beta \mid \alpha)[l, u]$, and $L \cup P^{\prime \prime} \models_{t i g h t}(\beta \mid \alpha)[r, s]$ imply $[l, u] \supseteq[r, s]$.

Proof of Theorem 3.7. Let $i \in\{0, \ldots, k\}$. Assume that every member of $P_{i}$ is tolerated under $L \cup\{\perp \Leftarrow \alpha\}$ by $P_{i} \cup \cdots \cup P_{k} \cup P^{\star}$. That is, for every $(\psi \mid \phi)[l, u] \in P_{i}$, there exists a model $\operatorname{Pr}$ of $L \cup P_{i} \cup \cdots \cup P_{k} \cup P^{\star}$ such 
that $\operatorname{Pr}(\alpha)=0$ and $\operatorname{Pr}(\phi)>0$. Thus, for every $P^{\prime}=\left\{\left(\psi_{1} \mid \phi_{1}\right)\left[l_{1}, u_{1}\right], \ldots,\left(\psi_{n} \mid \phi_{n}\right)\left[l_{n}, u_{n}\right]\right\} \subseteq P_{i} \cup \cdots \cup$ $P_{k} \cup P^{\star}$ such that $(\psi \mid \phi)[l, u] \in P^{\prime}$, a model $\operatorname{Pr}$ of $L \cup P^{\prime}$ exists such that $\operatorname{Pr}(\alpha)=0$ and $\operatorname{Pr}(\phi)>0$, and thus $\operatorname{Pr}\left(\phi_{1} \vee \cdots \vee \phi_{n} \vee \alpha\right)>0$. That is, no such $P^{\prime}$ belongs to $P_{\alpha}(K B)$. By induction on $i \in\{0, \ldots, k\}$, no $P^{\prime} \subseteq P$ with $P^{\prime} \cap\left(P_{0} \cup \cdots \cup P_{k}\right) \neq \emptyset$ belongs to $P_{\alpha}(K B)$.

Suppose that no member of $P^{\star}=\left\{\left(\psi_{1} \mid \phi_{1}\right)\left[l_{1}, u_{1}\right], \ldots,\left(\psi_{n} \mid \phi_{n}\right)\left[l_{n}, u_{n}\right]\right\}$ is tolerated under $L \cup\{\perp \Leftarrow \alpha\}$ by $P^{\star}$. It thus follows that no model $\operatorname{Pr}$ of $L \cup P^{\star}$ satisfies $\operatorname{Pr}\left(\phi_{1} \vee \cdots \vee \phi_{n}\right)>0$ and $\operatorname{Pr}(\alpha)=0$. That is, every model $\operatorname{Pr}$ of $L \cup P^{\star}$ with $\operatorname{Pr}\left(\phi_{1} \vee \cdots \vee \phi_{n} \vee \alpha\right)>0$ satisfies $\operatorname{Pr}(\alpha)>0$. Hence, $P^{\star}$ belongs to $P_{\alpha}(K B)$.

That is, (i) implies that every member of $P_{\alpha}(K B)$ is a subset of $P^{\star}$, and (ii) implies that $P^{\star}$ is in $P_{\alpha}(K B)$. In summary, $P^{\star}$ is the unique greatest element in $P_{\alpha}(K B)$.

Proof of Theorem 3.9. (a) Assume that $K B=(L, P)$ is g-coherent. By Theorem 3.2, for every nonempty $P_{n}=\left\{\left(\psi_{1} \mid \phi_{1}\right)\left[l_{1}, u_{1}\right], \ldots,\left(\psi_{n} \mid \phi_{n}\right)\left[l_{n}, u_{n}\right]\right\} \subseteq P$, there exists a model $\operatorname{Pr}$ of $L \cup P_{n}$ with $\operatorname{Pr}\left(\phi_{1} \vee \cdots \vee\right.$ $\left.\phi_{n}\right)>0$. In particular, $L \cup P$ has such a model $\operatorname{Pr}$. That is, $K B$ is satisfiable.

(b) Assume that $K B=(L, P)$ is strongly satisfiable. That is, a model $\operatorname{Pr}$ of $L \cup P$ exists such that $\operatorname{Pr}(\phi)>0$ for all $(\psi \mid \phi)[l, u] \in P$. Thus, for every nonempty $P_{n}=\left\{\left(\psi_{1} \mid \phi_{1}\right)\left[l_{1}, u_{1}\right], \ldots,\left(\psi_{n} \mid \phi_{n}\right)\left[l_{n}, u_{n}\right]\right\} \subseteq$ $P$, a model $\operatorname{Pr}$ of $L \cup P_{n}$ exists with $\operatorname{Pr}\left(\phi_{1}\right)>0$, and thus $\operatorname{Pr}\left(\phi_{1} \vee \cdots \vee \phi_{n}\right)>0$. By Theorem 3.2, $K B$ is g-coherent.

Proof of Theorem 3.11. Assume $K B \sim_{\text {tight }}(\beta \mid \alpha)[l, u]$ and $K B \models_{\text {tight }}(\beta \mid \alpha)[r, s]$. By Theorem 3.6 (b), it follows $\left(L, P^{\star}\right) \models_{\text {tight }}(\beta \mid \alpha)[l, u]$, where $P^{\star}$ is the unique greatest element in $P_{\alpha}(K B)$. Since $P^{\star} \subseteq P$, it thus follows $[l, u] \supseteq[r, s]$.

\section{Appendix B: Proofs for Section 4}

Proof of Theorem 4.4. The claim clearly holds for $P=\emptyset$. Assume now $P \neq \emptyset$.

$(\Rightarrow)$ Suppose that $K B$ is g-coherent. By Theorem $3.3\left(\right.$ b), there exists an ordered partition $\left(P_{0}, \ldots, P_{k}\right)$ of $P$ such that, for every $i \in\{0, \ldots, k\}$, each member of $P_{i}$ is tolerated under $L$ by $\bigcup_{j=i}^{k} P_{j}$. Define now the ranking $\sigma$ on $K B$ by $\sigma(F)=i$ for every $F \in P_{i}$ and $i \in\{0, \ldots, k\}$. We now show that $\sigma$ is admissible with $K B$. Assume the contrary. That is, there exists some $P^{\prime} \subseteq P$ that is under $L$ in conflict with some $F \in P$ such that $\sigma\left(F^{\prime}\right) \geq \sigma(F)$ for all $F^{\prime} \in P^{\prime}$. But, $F$ is tolerated under $L$ by $\left\{F^{\prime} \in P \mid \sigma\left(F^{\prime}\right) \geq \sigma(F)\right\} \supseteq P^{\prime}$, and thus also by $P^{\prime}$. This contradicts $P^{\prime}$ being under $L$ in conflict with $F$. Thus, $\sigma$ is admissible with $K B$.

$(\Leftarrow)$ Suppose there exists a ranking on $K B$ that is admissible with $K B$. Let $l$ be the cardinality of $\{\sigma(F) \mid F \in P\}$. We can assume $\{\sigma(F) \mid F \in K B\}=\{0, \ldots, k\}$, where $k=l-1$. For every $i \in\{0, \ldots, k\}$, define $P_{i}=\{F \in P \mid \sigma(F)=i\}$. Thus, $\left(P_{0}, \ldots, P_{k}\right)$ is an ordered partition of $P$ such that, for every $i \in$ $\{0, \ldots, k\}$, each $F \in P_{i}$ is tolerated under $L$ by $\bigcup_{j=i}^{k} P_{j}$. Thus, by Theorem 3.3 (b), $K B$ is g-coherent.

Proof of Theorem 4.5. Let $L$ be a set of logical constraints. We now show that $P^{\prime}=\left\{\left(\psi_{1}^{\prime} \mid \phi_{1}^{\prime}\right)[1,1], \ldots\right.$, $\left.\left(\psi_{m}^{\prime} \mid \phi_{m}^{\prime}\right)[1,1]\right\}$ tolerates $(\psi \mid \phi)[1,1]$ under $L$ iff $D^{\prime}=\left\{\psi_{1}^{\prime} \leftarrow \phi_{1}^{\prime}, \ldots, \psi_{m}^{\prime} \leftarrow \phi_{m}^{\prime}\right\}$ tolerates $\psi \leftarrow \phi$ under $L$. Assume first that $L \cup P^{\prime}$ has a model $\operatorname{Pr}$ such that $\operatorname{Pr} \models(\psi \mid \phi)[1,1]$ and $\operatorname{Pr}(\phi)>0$. Thus, there exists some $I \in \mathcal{I}_{\Phi}$ such that $I \models \phi$ and $\operatorname{Pr}(I)>0$. As $\operatorname{Pr}$ satisfies $L \cup P^{\prime}$ and $(\psi \mid \phi)[1,1]$, it follows that $I$ is a model of $L \cup D^{\prime}$ such that $I \models \phi \wedge \psi$. That is, $D^{\prime}$ tolerates $\psi \leftarrow \phi$ under $L$. Conversely, assume that $D^{\prime}$ tolerates $\psi \leftarrow \phi$ under $L$. That is, there exists a model $I^{\prime}$ of $L \cup D^{\prime}$ such that $I^{\prime} \models \phi \wedge \psi$. Hence, the probabilistic interpretation $\operatorname{Pr}$ defined by $\operatorname{Pr}\left(I^{\prime}\right)=1$ and $\operatorname{Pr}(I)=0$ for all other $I \in \mathcal{I}_{\Phi}$ is a model of $L \cup P^{\prime}$ such that $\operatorname{Pr} \models(\psi \mid \phi)[1,1]$ and $\operatorname{Pr}(\phi)=1$, and thus $\operatorname{Pr}(\phi)>0$. That is, $P^{\prime}$ tolerates $(\psi \mid \phi)[1,1]$ under $L$. Hence, toleration for conditional constraints of the form $(\psi \mid \phi)[1,1]$ coincides with toleration for defaults $\psi \leftarrow \phi$. Thus, the claim is immediate by Theorems 3.3 (b) and 4.2 (b). 
Proof of Theorem 4.9. Let $P^{\prime}=\left\{\left(\psi_{1}^{\prime} \mid \phi_{1}^{\prime}\right)[1,1], \ldots,\left(\psi_{m}^{\prime} \mid \phi_{m}^{\prime}\right)[1,1]\right\}$. We now show that $L \cup P^{\prime} \cup$ $\{(\beta \mid \alpha)[1,1]\}$ has a model $\operatorname{Pr}$ with $\operatorname{Pr}\left(\phi_{1}^{\prime} \vee \cdots \vee \phi_{m}^{\prime} \vee \alpha\right)>0$ iff there exists some $p \in(0,1]$ such that $L \cup P^{\prime} \cup\{(\beta \mid \alpha)[p, p]\}$ has a model $\operatorname{Pr}$ with $\operatorname{Pr}\left(\phi_{1}^{\prime} \vee \cdots \vee \phi_{m}^{\prime} \vee \alpha\right)>0$. Assume first that such $p \in(0,1]$ exists. Suppose $\operatorname{Pr}(\alpha)=0$. Hence, there exists some $I^{\prime} \in \mathcal{I}_{\Phi}$ with $I^{\prime} \models\left(\phi_{1}^{\prime} \vee \cdots \vee \phi_{m}^{\prime}\right) \wedge \neg \alpha$ and $\operatorname{Pr}\left(I^{\prime}\right)>0$. Define $\operatorname{Pr}^{\prime}$ by $\operatorname{Pr}^{\prime}\left(I^{\prime}\right)=1$ and by $\operatorname{Pr}^{\prime}(I)=0$ for all other $I \in \mathcal{I}_{\Phi}$. Then, $\operatorname{Pr}^{\prime} \models L \cup P^{\prime} \cup\{(\beta \mid \alpha)[1,1]\}$ and $\operatorname{Pr}^{\prime}\left(\phi_{1}^{\prime} \vee \cdots \vee \phi_{m}^{\prime} \vee \alpha\right)>0$. Assume next $\operatorname{Pr}(\alpha)>0$. Thus, there exists some $I^{\prime} \in \mathcal{I}_{\Phi}$ with $I^{\prime} \models \beta \wedge \alpha$ and $\operatorname{Pr}\left(I^{\prime}\right)>0$. Define $\operatorname{Pr}^{\prime}$ by $\operatorname{Pr}^{\prime}\left(I^{\prime}\right)=1$ and by $\operatorname{Pr}^{\prime}(I)=0$ for all other $I \in \mathcal{I}_{\Phi}$. Then, $\operatorname{Pr}^{\prime} \mid=L \cup P^{\prime} \cup$ $\{(\beta \mid \alpha)[1,1]\}$ and $\operatorname{Pr}^{\prime}\left(\phi_{1}^{\prime} \vee \cdots \vee \phi_{m}^{\prime} \vee \alpha\right)>0$. The converse trivially holds.

By Theorem 4.7, $K B \sim(\beta \mid \alpha)[1,1]$ iff $(L, P \cup\{(\beta \mid \alpha)[q, q]\})$ is not g-coherent for all $q \in[0,1)$, which is equivalent to $(L, P \cup\{(\neg \beta \mid \alpha)[p, p]\})$ not being g-coherent for all $p \in(0,1]$. By Theorem 3.2 and the above argumentation, the latter is equivalent to $(L, P \cup\{(\neg \beta \mid \alpha)[1,1]\})$ not being g-coherent. By Theorem 4.5, this is equivalent to $(L, D \cup\{\neg \beta \leftarrow \alpha\})$ being $p$-inconsistent. By Theorem 4.6, this is now equivalent to $\beta \leftarrow \alpha$ being a $p$-consequence of $(L, D)$.

\section{Appendix C: Proofs for Section 5}

Proof of Theorem 5.1. The statement follows immediately from Theorems 3.2 and 4.5 and the observation that $\left\{\psi_{1} \leftarrow \phi_{1}, \ldots, \psi_{n} \leftarrow \phi_{n}\right\}$ has a model $I$ such that $I \models L$ and $I \models \phi_{1} \vee \cdots \vee \phi_{n}$ iff $\left\{\left(\psi_{1} \mid \phi_{1}\right)[1,1], \ldots\right.$, $\left.\left(\psi_{n} \mid \phi_{n}\right)[1,1]\right\}$ has a model $\operatorname{Pr}$ such that $\operatorname{Pr} \models L$ and $\operatorname{Pr}\left(\phi_{1} \vee \cdots \vee \phi_{n}\right)>0$.

Proof of Lemma 5.2. Assume first that $L \models \neg \alpha$. Then, the statement is immediate by $D_{\alpha}(K B)=\emptyset=$ $P_{\alpha}\left(K B^{\prime}\right)$. Assume next that $L \not \models \neg \alpha$. Then, the statement follows from the observation that every model $I$ of $L \cup\left\{\psi_{1} \leftarrow \phi_{1}, \ldots, \psi_{n} \leftarrow \phi_{n}\right\}$ with $I \models \phi_{1} \vee \cdots \vee \phi_{n} \vee \alpha$ satisfies $I=\alpha$ iff every model $\operatorname{Pr}$ of $L \cup\left\{\left(\psi_{1} \mid \phi_{1}\right)[1,1], \ldots,\left(\psi_{n} \mid \phi_{n}\right)[1,1]\right\}$ with $\operatorname{Pr}\left(\phi_{1} \vee \cdots \vee \phi_{n} \vee \alpha\right)>0$ satisfies $\operatorname{Pr}(\alpha)>0$.

Proof of Theorem 5.3. The claim follows from Theorems 3.4, 4.5, and 4.9, Lemma 5.2, and the observation that $L \cup\left\{\psi_{1} \leftarrow \phi_{1}, \ldots, \psi_{n} \leftarrow \phi_{n}\right\} \models \beta \leftarrow \alpha$ iff $L \cup\left\{\left(\psi_{1} \mid \phi_{1}\right)[1,1], \ldots,\left(\psi_{n} \mid \phi_{n}\right)[1,1]\right\} \models(\beta \mid \alpha)[1,1]$.

Proof of Lemma 5.4. Immediate by Lemmata 3.5 and 5.2.

Proof of Theorem 5.5. $(\Rightarrow)$ Assume $K B \quad$-entails $\beta \leftarrow \alpha$. By Theorem 5.3, it follows $L \cup D^{\prime} \models \beta \Leftarrow \alpha$ for some $D^{\prime} \in D_{\alpha}(K B)$, and thus also $L \cup D^{\star} \models \beta \Leftarrow \alpha$.

$(\Leftarrow)$ Assume $L \cup D^{\star} \mid=\beta \Leftarrow \alpha$. Thus, by Theorem 5.3, KB $p$-entails $\beta \leftarrow \alpha$.

Proof of Theorem 5.6. By the proof of Theorem 4.5, toleration for conditional constraints of the form $(\psi \mid \phi)[1,1]$ coincides with toleration for defaults $\psi \leftarrow \phi$. Thus, the claim follows from Theorem 3.7 and Lemma 5.2.

\section{References}

[1] E. W. Adams. The Logic of Conditionals, volume 86 of Synthese Library. D. Reidel, Dordrecht, Netherlands, 1975.

[2] S. Amarger, D. Dubois, and H. Prade. Constraint propagation with imprecise conditional probabilities. In Proceedings UAI-91, pages 26-34. Morgan Kaufmann, 1991.

[3] S. Benferhat, D. Dubois, and H. Prade. Nonmonotonic reasoning, conditional objects and possibility theory. Artif. Intell., 92(1-2):259-276, 1997. 
[4] S. Benferhat, D. Dubois, and H. Prade. Possibilistic and standard probabilistic semantics of conditional knowledge bases. J. Logic Computat., 9(6):873-895, 1999.

[5] V. Biazzo and A. Gilio. A generalization of the fundamental theorem of de Finetti for imprecise conditional probability assessments. Int. J. Approx. Reasoning, 24(2-3):251-272, 2000.

[6] V. Biazzo, A. Gilio, T. Lukasiewicz, and G. Sanfilippo. Probabilistic logic under coherence: Complexity and algorithms. In Proceedings ISIPTA-01, pages 51-61, 2001.

[7] G. Boole. An Investigation of the Laws of Thought, on which are Founded the Mathematical Theories of Logic and Probabilities. Walton and Maberley, London, 1854. (reprint: Dover Publications, New York, 1958).

[8] G. Coletti. Coherent numerical and ordinal probabilistic assessments. IEEE Trans. Syst. Man Cybern., 24(12):1747-1754, 1994.

[9] G. Coletti and R. Scozzafava. Characterization of coherent conditional probabilities as a tool for their assessment and extension. Journal of Uncertainty, Fuzziness and Knowledge-based Systems, 4(2):103-127, 1996.

[10] G. Coletti and R. Scozzafava. Coherent upper and lower bayesian updating. In Proceedings ISIPTA-99, pages 101-110, 1999.

[11] G. Coletti and R. Scozzafava. Conditioning and inference in intelligent systems. Soft Computing, 3(3):118-130, 1999.

[12] D. Dubois and H. Prade. Possibilistic logic, preferential models, non-monotonicity and related issues. In Proceedings IJCAI-91, pages 419-424. Morgan Kaufmann, 1991.

[13] D. Dubois and H. Prade. Conditional objects as nonmonotonic consequence relationships. IEEE Trans. Syst. Man Cybern., 24(12):1724-1740, 1994.

[14] R. Fagin, J. Y. Halpern, and N. Megiddo. A logic for reasoning about probabilities. Inf. Comput., 87:78-128, 1990.

[15] A. M. Frisch and P. Haddawy. Anytime deduction for probabilistic logic. Artif. Intell., 69:93-122, 1994.

[16] D. M. Gabbay and P. Smets, editors. Handbook on Defeasible Reasoning and Uncertainty Management Systems. Kluwer Academic, Dordrecht, Netherlands, 1998.

[17] H. Geffner. Default Reasoning: Causal and Conditional Theories. MIT Press, 1992.

[18] H. Geffner. High probabilities, model preference and default arguments. Mind and Machines, 2:51-70, 1992.

[19] A. Gilio. Algorithms for precise and imprecise conditional probability assessments. In G. Coletti, D. Dubois, and R. Scozzafava, editors, Mathematical Models for Handling Partial Knowledge in Artificial Intelligence, pages 231-254. Plenum Press, New York, 1995.

[20] A. Gilio. Probabilistic consistency of conditional probability bounds. In Advances in Intelligent Computing, volume 945 of $L N C S$, pages 200-209. Springer, 1995.

[21] A. Gilio. Probabilistic reasoning under coherence in System P. Ann. Math. Artif. Intell., 34(1-3):5-34, 2002.

[22] A. Gilio and R. Scozzafava. Conditional events in probability assessment and revision. IEEE Trans. Syst. Man Cybern., 24(12):1741-1746, 1994.

[23] M. Goldszmidt and J. Pearl. On the consistency of defeasible databases. Artif. Intell., 52(2):121-149, 1991.

[24] M. Goldszmidt and J. Pearl. Rank-based systems: A simple approach to belief revision, belief update and reasoning about evidence and actions. In Proceedings KR-92, pages 661-672. Morgan Kaufmann, 1992.

[25] S. Kraus, D. Lehmann, and M. Magidor. Nonmonotonic reasoning, preferential models and cumulative logics. Artif. Intell., 14(1):167-207, 1990. 
[26] D. Lehmann. Another perspective on default reasoning. Ann. Math. Artif. Intell., 15(1):61-82, 1995.

[27] T. Lukasiewicz. Local probabilistic deduction from taxonomic and probabilistic knowledge-bases over conjunctive events. Int. J. Approx. Reasoning, 21(1):23-61, 1999.

[28] T. Lukasiewicz. Probabilistic deduction with conditional constraints over basic events. J. Artif. Intell. Res., 10:199-241, 1999.

[29] T. Lukasiewicz. Probabilistic logic programming with conditional constraints. ACM Trans. Computat. Logic, 2(3):289-339, 2001.

[30] T. Lukasiewicz. Nonmonotonic probabilistic logics between model-theoretic probabilistic logic and probabilistic logic under coherence. In Proceedings of the 9th International Workshop on Non-Monotonic Reasoning (NMR2002), pages 265-274, 2002.

[31] T. Lukasiewicz. Probabilistic default reasoning with conditional constraints. Ann. Math. Artif. Intell., 34(13):35-88, 2002.

[32] N. J. Nilsson. Probabilistic logic. Artif. Intell., 28:71-88, 1986.

[33] J. Pearl. Probabilistic Reasoning in Intelligent Systems: Networks of Plausible Inference. Morgan Kaufmann, San Mateo, CA, 1988.

[34] J. Pearl. Probabilistic semantics for nonmontonic reasoning: A survey. In Proceedings KR-89, pages 505-516. Morgan Kaufmann, 1989.

[35] J. Pearl. System Z: A natural ordering of defaults with tractable applications to default reasoning. In Proceedings TARK-90, pages 121-135. Morgan Kaufmann, 1990.

[36] R. Pelessoni and P. Vicig. A consistency problem for imprecise conditional probability assessments. In Proceedings IPMU-98, pages 1478-1485, 1998.

[37] R. Scozzafava. Subjective conditional probability and coherence principles for handling partial information. Mathware Soft Comput., 3(1):183-192, 1996.

[38] Y. Shoham. A semantical approach to nonmonotonic logics. In Proceedings of the 2nd IEEE Symposium on Logic in Computer Science, pages 275-279, 1987.

[39] P. Snow. Diverse confidence levels in a probabilistic semantics for conditional logics. Artif. Intell., 113:269-279, 1999.

[40] W. Spohn. Ordinal conditional functions: A dynamic theory of epistemic states. In W. Harper and B. Skyrms, editors, Causation in Decision, Belief Change, and Statistics, volume 2, pages 105-134. Reidel, Dordrecht, Netherlands, 1988.

[41] P. Walley. Statistical Reasoning with Imprecise Probabilities. Chapman and Hall, 1991. 